\title{
Higher-charge three-dimensional compact lattice Abelian-Higgs models
}

\author{
Claudio Bonati, ${ }^{1}$ Andrea Pelissetto, ${ }^{2}$ and Ettore Vicari $\odot^{1}$ \\ ${ }^{1}$ Dipartimento di Fisica dell'Università di Pisa and INFN, Largo Pontecorvo 3, I-56127 Pisa, Italy \\ ${ }^{2}$ Dipartimento di Fisica dell'Università di Roma Sapienza and INFN, Sezione di Roma I, P.le A. Moro 2, I-00185 Roma, Italy
}

(Received 12 November 2020; accepted 14 December 2020; published 30 December 2020)

\begin{abstract}
We consider three-dimensional higher-charge multicomponent lattice Abelian-Higgs (AH) models, in which a compact U(1) gauge field is coupled to an $N$-component complex scalar field with integer charge $q$, so that they have local $\mathrm{U}(1)$ and global $\mathrm{SU}(N)$ symmetries. We discuss the dependence of the phase diagram, and the nature of the phase transitions, on the charge $q$ of the scalar field and the number $N \geqslant 2$ of components. We argue that the phase diagram of higher-charge models presents three different phases, related to the condensation of gaugeinvariant bilinear scalar fields breaking the global $\mathrm{SU}(N)$ symmetry, and to the confinement and deconfinement of external charge-one particles. The transition lines separating the different phases show different features, which also depend on the number $N$ of components. Therefore, the phase diagram of higher-charge models substantially differs from that of unit-charge models, which undergo only transitions driven by the breaking of the global $\mathrm{SU}(N)$ symmetry, while the gauge correlations do not play any relevant role. We support the conjectured scenario with numerical results, based on finite-size scaling analyses of Monte Carlo simuations for doubly charged unit-length scalar fields with small and large number of components, i.e., $N=2$ and $N=25$.
\end{abstract}

DOI: 10.1103/PhysRevE.102.062151

\section{INTRODUCTION}

Abelian U(1) gauge theories with multicomponent scalar fields, characterized by a global $\mathrm{SU}(N)$ symmetry, emerge as effective theories in many different physical contexts [1-13]. In particular, they provide an effective description of deconfined quantum critical points [14], for example, of the Néel to valence-bond-solid transition in two-dimensional antiferromagnetic SU(2) quantum systems [15-22]. These quantum models and their classical counterparts have been extensively studied to understand their different phases and the nature of their phase transitions. A crucial role is played by topological aspects, like the Berry phase or the compact/noncompact nature of the gauge fields. For example, the critical behavior of the lattice $\mathrm{CP}^{N-1}$ model, which is the simplest classical model with $U(1)$ gauge symmetry, depends on the presence/absence of topological defects [23-27], such as monopoles, both for $N=2$ and large values of $N$. Analogous differences emerge in the behavior of compact and noncompact lattice formulations of scalar electrodynamics, i.e., of the multicomponent Abelian-Higgs model; see, e.g., Refs. [12,14-22,2745].

In this paper we consider three-dimensional (3D) highercharge multicomponent lattice Abelian-Higgs (AH) models. In these models a compact $\mathrm{U}(1)$ gauge field is coupled to an $N$-component complex scalar field with integer charge $q>1$, so that they are invariant under local $\mathrm{U}(1)$ and global $\mathrm{SU}(N)$ transformations. We study the dependence of the thermodynamic properties, such as the phase diagram and the nature of the phase transitions, on the value of the charge $q$. Our work extends previous studies of the compact lattice $\mathrm{AH}$ model with a single $(N=1)$ higher-charge complex scalar field [46-52] to multicomponent $N \geqslant 2$ theories. For $N=1$ and $q \geqslant 2$, the phase diagram is characterized by two phases, that are distinguished by the confinement and deconfinement of single-charge external particles. In this case, the Wilson loops associated with charge-one particles can be considered as the order parameter of the transitions, which separate the confined high-temperature phase, in which Wilson loops obey the area law, from the deconfined phase. As we shall see, for $N \geqslant 2$ the confinement and deconfinement of charge-one external sources also plays an important role in determining the phase diagram. However, there are also new features related to the breaking of the global $\mathrm{SU}(N)$ symmetry.

The phase diagram of the multicomponent compact $\mathrm{AH}$ model with $q \geqslant 2$ also presents notable differences with respect to that of the same model with $q=1$. For $N \geqslant 2$ and $q=1$ (this has been explicitly verified in the London limit in which the scalar fields have unit length) there are only two phases, that can be characterized by using a gauge-invariant scalar-field order parameter, while gauge fluctuations do not play any relevant role [44]. In particular, for $N=2$ the transition line between the high- and the low-temperature phase shows continuous transitions belonging to the $\mathrm{CP}^{1}-$ equivalently, $\mathrm{O}(3)$ vector-universality class.

We argue, and present numerical results to support our arguments, that, for $q \geqslant 2$ and $N \geqslant 2$, the model with compact gauge fields and unit-length complex scalar fields (London limit) shows three phases, as sketched in Fig. 1. They are characterized by the behavior of the gauge-invariant correlations of the scalar fields, which may give rise to the breaking of the $\mathrm{SU}(N)$ global symmetry, and by the confinement and deconfinement of charge-one external particles, that is signalled by the large-size behavior of the Wilson loops of the gauge 


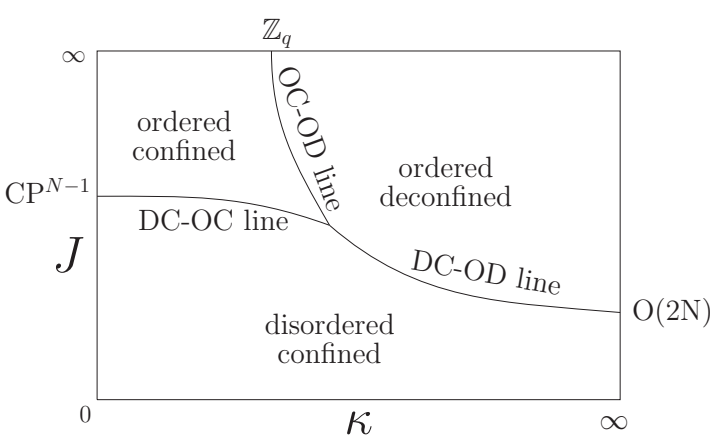

FIG. 1. Sketch of the $J-\kappa$ phase diagram of the 3D multicomponent lattice Abelian-Higgs model, in which a compact U(1) gauge field is coupled to an $N$-component unit-length complex scalar field with charge $q \geqslant 2$, for generic $N \geqslant 2$. The Hamiltonian parameter $J$ is associated with the kinetic gauge-invariant term of the scalar field, while $\kappa$ represents the inverse gauge coupling. See text for a description of the various phases and transition lines. We also report the models emerging in some limiting cases: the $\mathrm{CP}^{N-1}$ model for $\kappa=0$, the $\mathrm{O}(2 N)$ vector model for $\kappa \rightarrow \infty$, and the lattice $\mathbb{Z}_{q}$ gauge model for $J \rightarrow \infty$.

fields. As shown in Fig. 1, for small $J$ and any $\kappa \geqslant 0$, there is a phase in which scalar-field correlations are disordered and single-charge particles are confined (the Wilson loop obeys the area law). For large values of $J$ (low-temperature region) scalar correlations are ordered and the $\mathrm{SU}(N)$ symmetry is broken. Two phases occur here: for small $\kappa$, single-charge particles are confined, while they are deconfined for large $\kappa$. These phases are separated by three transition lines meeting at a multicritical point: the DC-OD transition line between the disordered and confined (DC) and the ordered and deconfined (OD) phases, the DC-OC line between the disordered and confined and the ordered and confined (OC) phases, and the OC-OD line between the ordered and confined and ordered and deconfined phases.

The three transition lines have different features, since they are associated with different phases. Moreover, their nature crucially depends on the number $N$ of components. In particular, for $q=2$ and $N=2$, we provide evidence that the transitions along the DC-OC and OC-OD lines are continuous, belonging to the $\mathrm{O}(3)$ vector and Ising universality class, respectively. The transitions along the DC-OD line are of first order. For large values of $N$ we expect a different behavior along the DC-OC and DC-OD lines. The transitions along the DC-OC line are expected to be first order. As we shall see, our numerical results for $q=2$ and $N=25$ provide evidence of continuous transitions along the DC-OD line.

We note that the different qualitative behavior of the models with $q=1$ and $q \geqslant 2$ is a specific feature of the compact formulation of the AH theory. Indeed, in the AH model with noncompact gauge fields a change of the charge of the scalar field is equivalent to a change of the strength of the gauge coupling. Therefore, apart from a trivial rescaling of the gauge coupling, the phase diagram is the same. We also note that the noncompact formulation of the AH theory should be recovered in the $q \rightarrow \infty$ limit of the compact formulation, with an appropriate correspondence of the gauge couplings.
The paper is organized as follows. In Sec. II we introduce the lattice AH model with an $N$-component scalar field of generic charge $q$, and define the relevant observables that characterize the phase transitions. In Sec. III we present the possible scenarios for the phase diagram and for the nature of the transition lines. Section IV presents our numerical results for $q=2$ : we report FSS analyses [53-56] of the Monte Carlo (MC) results for $N=2$ and $N=25$, which allow us to determine the phase diagram of the model and to characterize the different transition lines. In Sec. V we discuss the role that monopoles play in the compact model with $q \geqslant 2$. Finally, we draw our conclusions in Sec. VI.

\section{THE HIGHER-CHARGE LATTICE AH MODEL}

\section{A. The model}

We consider a three-dimensional lattice AH model, in which the scalar field $z_{x}$ is a complex $N$-component unit vector $\left(\bar{z}_{x} \cdot z_{x}=1\right)$ of integer charge $q$ defined on the sites $\boldsymbol{x}$ of a cubic lattice. For the gauge fields, we use the compact Wilson formulation, associating complex variables $\lambda_{x, \mu}$ with $\left|\lambda_{x, \mu}\right|=1$ to each link connecting the site $\boldsymbol{x}$ with the site $\boldsymbol{x}+\hat{\mu}$, where $\hat{\mu}=\hat{1}, \hat{2}, \hat{3}$ are unit vectors along the lattice directions. The Hamiltonian reads

$$
H=J N H_{z}+\kappa H_{g} .
$$

The first term is the interaction term for the scalar fields of charge $q$ :

$$
H_{z}=-\sum_{x, \mu}\left(\bar{z}_{x} \cdot \lambda_{x, \mu}^{q} z_{x+\hat{\mu}}+\text { c.c. }\right),
$$

where the sum is over all lattice links of the cubic lattice. The second term is the usual Wilson Hamiltonian for a U(1) gauge field:

$$
H_{g}=-\sum_{\boldsymbol{x}, \mu<\nu}\left(\Pi_{\boldsymbol{x}, \mu \nu}+\text { c.c. }\right),
$$

where the sum is over the lattice plaquettes and $\Pi_{x, \mu \nu}$ is the field strength associated with each plaquette,

$$
\Pi_{x, \mu \nu}=\lambda_{x, \mu} \lambda_{x+\hat{\mu}, \nu} \bar{\lambda}_{x+\hat{v}, \mu} \bar{\lambda}_{x, \nu}
$$

For any integer $q$ the model is invariant under the global $\mathrm{SU}(N)$ transformations $z_{x} \rightarrow U z_{x}, \lambda_{x, \mu} \rightarrow \lambda_{x, \mu}$, where $U \in$ $\mathrm{SU}(N)$, and under the local $\mathrm{U}(1)$ transformations $z_{x} \rightarrow$ $e^{i q \phi_{x}} z_{x}, \lambda_{x, \mu} \rightarrow e^{i\left(\phi_{x}-\phi_{x+\hat{\mu}}\right)} \lambda_{x, \mu}$, where $\phi_{x}$ is a site-dependent phase. For $q>1$ there are additional global symmetries that only involve the gauge field. If we consider the sites $\boldsymbol{y}$ that belong to a given plane-for definiteness consider a plane orthogonal to the direction $\hat{1}-$ then the Hamiltonian is invariant under the transformation $\lambda_{y, 1} \rightarrow \alpha \lambda_{y, 1}$ on all these sites, where $\alpha$ satisfies $\alpha^{q}=1$. The $\mathbb{Z}_{q}$ symmetry is the analog of the center symmetry that is present in pure lattice gauge theories. Its spontaneous breaking signals the deconfinement of the single-charge sources. The partition function of the system reads

$$
Z=\sum_{\{z\},\{\lambda\}} e^{-\beta H}
$$


In the following we rescale $J$ and $\kappa$ by $\beta$, thus formally setting $\beta=1$. The numerical FSS analyses reported in Sec. IV will consider cubic $L^{3}$ lattices with periodic boundary conditions.

\section{B. Observables}

To characterize phase transitions associated with the breaking of the $\mathrm{SU}(N)$ symmetry, we consider correlations of the gauge-invariant Hermitean operator

$$
Q_{x}^{a b}=\bar{z}_{x}^{a} z_{x}^{b}-\frac{1}{N} \delta^{a b} .
$$

Its two-point correlation function is defined as

$$
G(\boldsymbol{x}-\boldsymbol{y})=\left\langle\operatorname{Tr} Q_{x} Q_{y}\right\rangle,
$$

where the translation invariance of the system has been taken into account. The susceptibility and the correlation length are defined as $\chi=\sum_{x} G(\boldsymbol{x})$ and

$$
\xi^{2} \equiv \frac{1}{4 \sin ^{2}(\pi / L)} \frac{\widetilde{G}(\mathbf{0})-\widetilde{G}\left(\boldsymbol{p}_{m}\right)}{\widetilde{G}\left(\boldsymbol{p}_{m}\right)},
$$

where $\widetilde{G}(\boldsymbol{p})=\sum_{x} e^{i \boldsymbol{p} \cdot \boldsymbol{x}} G(\boldsymbol{x})$ is the Fourier transform of $G(\boldsymbol{x})$, and $\boldsymbol{p}_{m}=(2 \pi / L, 0,0)$ is the minimum nonzero lattice momentum.

In our analysis we will consider renormalization-group (RG) invariant quantities, such as $R_{\xi}=\xi / L$ and the Binder parameter

$$
U=\frac{\left\langle\mu_{2}^{2}\right\rangle}{\left\langle\mu_{2}\right\rangle^{2}}, \quad \mu_{2}=\sum_{\boldsymbol{x}, \boldsymbol{y}} \operatorname{Tr} Q_{x} Q_{\boldsymbol{y}} .
$$

We also consider the energy-related observables

$$
\begin{array}{ll}
E_{z}=-\frac{1}{2 V}\left\langle H_{z}\right\rangle, & C_{z}=\frac{1}{4 V}\left(\left\langle H_{z}^{2}\right\rangle-\left\langle H_{z}\right\rangle^{2}\right), \\
E_{g}=-\frac{1}{2 V}\left\langle H_{g}\right\rangle, & C_{g}=\frac{1}{4 V}\left(\left\langle H_{g}^{2}\right\rangle-\left\langle H_{g}\right\rangle^{2}\right),
\end{array}
$$

where $V=L^{3}$. They correspond to the energy density and to the specific heat associated with the scalar and gauge part of the Hamiltonian, respectively. The normalizations have been chosen so that $E_{z}$ and $E_{g}$ converge to 3 in the ordered limit $J, \kappa \rightarrow \infty$. We also consider the third cumulant

$$
H_{g 3}=\left\langle\left(H_{g}-\left\langle H_{g}\right\rangle\right)^{3}\right\rangle .
$$

We consider correlation functions of plaquette operators, such as the connected correlation function

$$
G_{\pi}(\boldsymbol{x}-\boldsymbol{y})=\left\langle\Sigma_{\boldsymbol{x}} \Sigma_{\boldsymbol{y}}\right\rangle_{c}, \quad \Sigma_{\boldsymbol{x}}=\operatorname{Re} \sum_{\mu<\nu} \Pi_{\boldsymbol{x}, \mu \nu} .
$$

Note that $\sum_{x} G_{\pi}(\boldsymbol{x})=C_{g}$, cf. Eq. (11). Using $G_{\pi}(\boldsymbol{x})$ one can also define a correlation length $\xi_{\pi}$ as in Eq. (8).

We finally define a field-strength correlation function. For definiteness, we select the $x$ direction and define $(x, y, z$ are the coordinates of each lattice point)

$$
n_{x}=\operatorname{Im}\left(\sum_{y z} \Pi_{(x, y, z), 23}\right) .
$$

Then we consider a lattice plane orthogonal to the unit vector $\hat{1}$ and sum over all plaquettes that belong to the plane. When the gauge fields are close to $1, n_{x}$ is essentially the sum of the field strengths on the plane. Then, we define a correlation function

$$
G_{F}\left(x_{1}-x_{2}\right)=\left\langle n_{x_{1}} n_{x_{2}}\right\rangle,
$$

and a correlation length $\xi_{F}$ using Eq. (8). Of course, one can define an analogous correlation function $G_{F}(x)$ by considering the $y$ or the $z$ direction. In our simulation, we compute $G_{F}(x)$ by averaging the correlation function over the three lattice directions.

\section{THE PHASE DIAGRAM}

\section{A. Some limiting cases}

To understand the phase diagram of the model, it is useful to consider some particular cases, in which the thermodynamic behavior is already known. No transitions are expected along the $J=0$ line, while transitions occur along the $\kappa=0$, the $J=\infty$, and the $\kappa=\infty$ lines.

\section{Phase diagram along the $\kappa=0$ line}

For $\kappa=0$ the model is equivalent to a lattice formulation of the $\mathrm{CP}^{N-1}$ models with explicit lattice gauge variables [57]. Indeed, for $\kappa=0$ the charge $q$ does not play any role: one can redefine $\lambda^{q} \rightarrow \lambda^{\prime}$, without changing the model. Its phase diagram has two phases separated by a finite-temperature transition, where the order parameter is the gauge-invariant bilinear operator defined in Eq. (6). Some estimates of the transition point $J_{c}$ have been obtained in Refs. [57,58], and summarized in Ref. [45]. For $N=2$ the transition is continuous, belonging to the $\mathrm{O}(3)$ vector universality class (accurate estimates of the corresponding critical exponents can be found in Refs. [56,59-63]). For $N \geqslant 3$ it is instead of first order: It is weak for $N=3$ [57] and becomes stronger and stronger with increasing $N$ [58]. We expect that nature of the transitions to persist for finite, sufficiently small values of $\kappa$.

\section{Phase diagram along the $J=\infty$ line}

For $J \rightarrow \infty$ the relevant configurations are those that minimize the spin Hamiltonian $H_{z}$ defined in Eq. (2). In the lowest-energy configuration the fields $z_{x}$ satisfy

$$
z_{x}=\lambda_{x, \mu}^{q} z_{x+\hat{\mu}}
$$

Iterating this relation along a plaquette, we obtain

$$
\Pi_{\boldsymbol{x}, \mu \nu}^{q}=1
$$

on all plaquettes. A similar result holds for the product of the fields $\lambda_{x, \mu}^{q}$ along any topologically nontrivial lattice loop. Therefore, modulo gauge transformations, we can set $\lambda_{x, \mu}^{q}=$ 1 on any lattice link. This condition implies

$$
\lambda_{x, \mu}=\exp (2 \pi i n / q), \quad n=0, \ldots, q-1 .
$$

Thus, in the $J \rightarrow \infty$ limit we obtain a lattice $\mathbb{Z}_{q}$ gauge theory, in which the spin variables are associated with the links of the lattice. These models can be related by duality to $q$ state clock spin models [48], characterized by a global $\mathbb{Z}_{q}$ symmetry. For $q=2$, the $q$-state clock model is equivalent to the standard Ising model and thus we expect an Ising transition for $J=\infty$. For $q=3$, the $q$-state clock model is 
equivalent to a three-state Potts model, which can only undergo first-order transitions. For larger values of $q$, we expect a continuous transition. It belongs to the Ising universality class for $q=4$ [64], and to the 3D $X Y$ universality class for $q \geqslant 5$ [64-66]. It is important to note that, for $q=2$, duality allows us to map the $\mathbb{Z}_{2}$ gauge Hamiltonian Eq. (3) onto the usual nearest-neighbor Ising model. This allows us to predict $\kappa_{c}=\frac{1}{4} \ln \operatorname{coth} \beta_{I, c}$, where $\beta_{I, c}$ is the inverse temperature of the Ising model. Using [67] $\beta_{I, c}=0.221654626(5)$, we obtain $\kappa_{c}=0.380706646(6)$.

\section{Phase diagram along the $\kappa=\infty$ line}

For $\kappa \rightarrow \infty$ the gauge degrees of freedom are frozen and we can set $\lambda_{x, \mu}=1$ modulo gauge transformations. In this limit the model is therefore equivalent to the $\mathrm{O}(2 N)$ vector model, which undergoes a continuous transitions for any $N$. The same occurs in the standard lattice $q=1$ AH models with compact and noncompact gauge fields; see, e.g., Refs. [44,45]. Estimates of the critical values $J_{c}$ along the $\kappa=\infty$ line are summarized in Ref. [45]. They are obtained from the results reported in Refs. [58,68-71]. The RG analysis of the continuum AH field theory, see, e.g., Refs. [44,45], predicts that gauge modes are a relevant perturbation of the $\mathrm{O}(2 \mathrm{~N})$ fixed point. Therefore, the DC-OD transitions do not belong to the $\mathrm{O}(2 N)$ vector universality class. However, the $\mathrm{O}(2 N)$ continuous transition for $\kappa=\infty$ gives rise to crossover phenomena for large values of $\kappa$.

\section{B. Transition lines}

On the basis of the above considerations, the most natural phase diagram is the one reported in Fig. 1. There are three different phases, characterized by the behavior of the spin and gauge correlations. The spin order parameter is the bilinear scalar operator $Q_{x}$ defined in Eq. (6), that signals the breaking of the global $\mathrm{SU}(N)$ symmetry. The behavior of the gauge modes can be understood by looking at the behavior of the charge-one Wilson loops,

$$
W_{\mathcal{C}}=\prod_{\ell \in \mathcal{C}} \lambda_{\ell},
$$

where $\mathcal{C}$ is a closed lattice loop. Depending on the phase, the Wilson loop may or may not obey the area law; correspondingly charge-one sources may be confined or deconfined.

Using these two order parameters, we can characterize the different phases. For small $J$ and any $\kappa$, there is a hightemperature disordered-confined (DC) phase with disordered scalar-field correlations and confined charge-one particles. For large $J$ there are two phases, in which spin correlations are ordered and the global $\mathrm{SU}(N)$ symmetry is broken. They differ in the behavior of the gauge correlations: for small $\kappa$ charge-one sources are confined, while for large $\kappa$ they are deconfined. These phases are separated by three distinct transition lines, that presumably meet at a multicritical point (MCP) at $\left(\kappa_{m}, J_{m}\right)$; see Fig. 1.

Note that the value of the charge plays here a crucial role. For the unit-charge theory, the area law never holds, as soon as the scalar interaction is turned on, a phenomenon known as screening. For instance, for $J$ small there is always a contribu- tion to $W_{\mathcal{C}}$ of order $J^{p_{\mathcal{C}}}$, where $p_{\mathcal{C}}$ is the length of the Wilson loop. These contributions are absent for any $q \geqslant 2$.

The above predictions strictly apply to the lattice $\mathrm{AH}$ model in the London limit, in which the modulus $\left|z_{x}\right|$ is kept fixed. However, we believe that the same phases and transitions occur in more general models in which the constraint is relaxed.

\section{The $O C-O D$ transition line}

The nature of the OC-OD transition line is expected to be independent of the number of components, at least for sufficiently large values of $J$. For $q=2$ it is continuous, belonging to the Ising universality class, for any $J>J^{*}$, where $J^{*}$ may coincide with the position of the MCP, i.e., $J^{*} \geqslant J_{m}$. Along this transition line the scalar-field fluctuations are expected to be irrelevant, acting as spectators. For $q=3$ the first-order nature of the transition at $J=\infty$ is expected to persist for finite values of $J$. The transition line is expected to be continuous for $q \geqslant 4$ and to belong to the $X Y$ universality class for $q \geqslant 5$.

\section{The DC-OC transition line}

The transitions along the DC-OC line are expected to have the same nature as the $\kappa=0$ transition, at least for $\kappa<\kappa^{*}$, where $\kappa^{*}$ must satisfy $\kappa^{*} \leqslant \kappa_{m}$. As we said, we do not expect the addition of $H_{g}$ for small $\kappa$ to change the nature of the transition. Therefore, as it occurs for $\kappa=0$, we expect gauge modes to be irrelevant. Thus, the transitions for $N=2$ should be continuous and belong to the $\mathrm{O}(3)$ vector universality class, while they should be of first order for $N \geqslant 3$. As in the $\mathrm{CP}^{N-1}$ model $(\kappa=0)$, the DC-OC transition line is characterized by the condensation of the gauge-invariant bilinear operator Eq. (6).

\section{The $D C-O D$ transition line}

The nature of the DC-OD transition line is less clear. The bilinear operator Eq. (6) is again expected to be an appropriate order parameter. Moreover, as $J$ increases across the transition line, we also expect deconfinement: the area law ceases to hold in the OD phase. On the basis of the numerical results reported in Sec. IV, we shall argue that the behavior along the DC-OD transition line is controlled by the stable fixed point of the continuum AH field theory, whose Lagrangian reads

$$
\mathcal{L}=\left|D_{\mu} \boldsymbol{\Phi}\right|^{2}+r \boldsymbol{\Phi}^{*} \boldsymbol{\Phi}+\frac{1}{6} u\left(\boldsymbol{\Phi}^{*} \boldsymbol{\Phi}\right)^{2}+\frac{1}{4 g^{2}} F_{\mu \nu}^{2},
$$

where $\Phi$ is a $N$-component complex scalar field, $F_{\mu \nu} \equiv$ $\partial_{\mu} A_{\nu}-\partial_{\nu} A_{\mu}$, and $D_{\mu} \equiv \partial_{\mu}+i A_{\mu}$. This is analogous to what occurs along the Coulomb-Higgs line of the lattice AH model with unit-charge spin fields and noncompact gauge fields [45]. We recall that the $\mathrm{RG}$ flow of the $\mathrm{AH}$ field theory predicts that continuous transitions may be observed only for $N>N_{c}[1,72,73]$, with $4<N_{c}<10$ in three dimensions $[45,73]$.

\section{Nature of the bicritical point for $q=2$ and $N=2$}

As discussed above, the phase diagram of the lattice $\mathrm{AH}$ model with charge $q=2$ is characterized by three transition lines meeting at a MCP, which is usually called bicritical 
point [74-77]. To discuss its nature, it is crucial to identify the relevant critical modes. Let us focus on the particular case $N=2$, where we expect continuous transitions along the DC-OC line with an $\mathrm{O}(3)$ scalar order parameter, and continuous transitions along the OC-OD line with an Ising order parameter. Therefore, the nature of the MCP is determined by the competition of the effective order parameters appropriate for the continuous DC-OC and OC-OD transitions. This hypothesis is quite reasonable, as the two transition lines are associated with different degrees of freedom: transitions along the DC-OC line are only driven by the condensation of bilinear scalar fields, while gauge fields drive the transitions along the OC-OD line. The nature of the bicritical point can be investigated within the Landau-Ginzburg-Wilson (LGW) framework. One considers the most general scalar $\Phi^{4}$ theory that is invariant under $\mathrm{O}(3) \oplus \mathbb{Z}_{2}$ transformations [74-77], i.e.,

$$
\begin{aligned}
\mathcal{H}_{\mathrm{LGW}}= & \frac{1}{2}\left[\left(\partial_{\mu} \psi\right)^{2}+\left(\partial_{\mu} \varphi\right)^{2}\right]+\frac{1}{2}\left(r_{\phi} \psi^{2}+r_{\varphi} \varphi^{2}\right) \\
& +\frac{1}{4 !}\left[u_{\phi}\left(\psi^{2}\right)^{2}+u_{\varphi}\left(\varphi^{2}\right)^{2}+2 w \psi^{2} \varphi^{2}\right],
\end{aligned}
$$

where the three-component field $\psi$ and the one-component field $\varphi$ are the coarse-grained $\mathrm{O}(3)$ and $\mathbb{Z}_{2}$ order parameters. This LGW field theory has been extensively studied in Refs. [74-77]. In the mean-field approximation [74-76], the model admits a phase diagram analogous to that sketched in Fig. 1, with a bicritical point, where two continuous transition lines (belonging to the Heisenberg and Ising universality classes) and a first-order transition line meet. The nature of the MCP depends on the stability of the fixed points of the RG flow of the $\Phi^{4}$ theory Eq. (21). A continuous transition is possible only if the RG flow admits a stable fixed point.

The RG flow of the model was studied in Ref. [77]. Results were not conclusive for the particular $\mathrm{O}(3) \oplus \mathbb{Z}_{2}$ LGW theory considered here, leaving open the possibility of observing a bicritical continuous transition controlled by the so-called biconical fixed point. If such fixed point is stable, then the transitions along the DC-OC and OC-OD lines may be continuous up to the the MCP at $\left(\kappa_{m}, J_{m}\right)$. In the opposite case, the continuous transitions along the two lines turn into first-order transitions before reaching the MCP. It is important to note that, independently of the stability of the biconical fixed point, the LGW $\Phi^{4}$ theory predicts the DC-OD transition line to be of first order close to the MCP. As $\kappa$ is increased along this line, the first-order transition should become weaker and weaker (the latent heat decreases) as the $\mathrm{O}(4)$ continuous transition at $\kappa=\infty$ is approached, with substantial crossover phenomena occurring for large values of $\kappa$. Note that, a priori we cannot exclude that the first-order transitions turn into continuous ones for finite values of $\kappa$.

\section{NUMERICAL RESULTS FOR THE DOUBLY CHARGED MODEL}

We present a numerical study based on MC simulations of the doubly charged lattice $\mathrm{AH}$ model with $N=2$ and $N=$ 25 . We consider cubic lattices of linear size $L$ with periodic boundary conditions. As in our previous work [44,57], we perform microcanonical and Metropolis updates of the scalar fields. For the gauge field $\lambda_{x, \mu}$ we only perform Metropolis
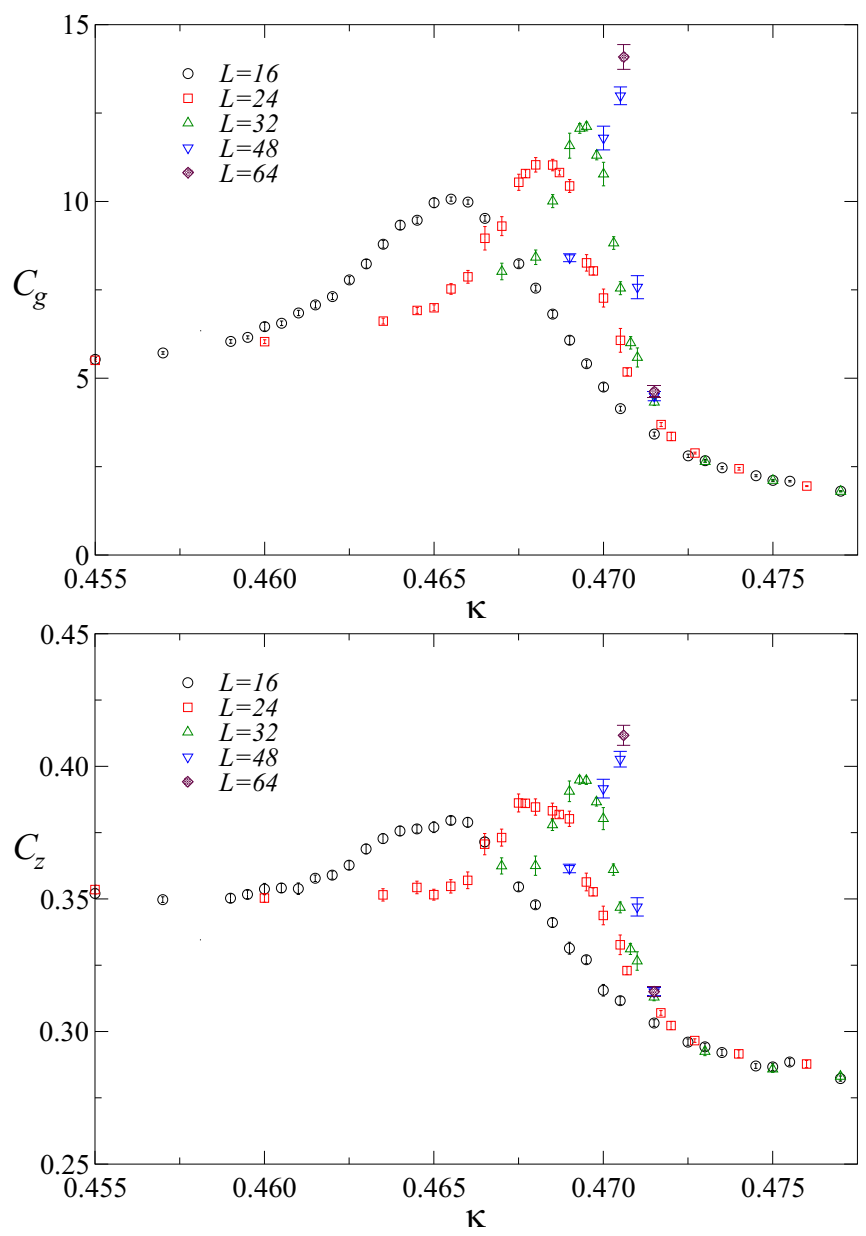

FIG. 2. Plot of the specific heats $C_{g}$ and $C_{z}$ as a function of $\kappa$ for $J=0.8$ and $N=2$.

updates: we propose $\lambda_{x, \mu} \rightarrow e^{i \varphi} \lambda_{x, \mu}$, choosing $\varphi$ either uniformly around 0 (more precisely, in $0 \leqslant|\varphi| \leqslant a$, where $a$ is chosen to obtain an average acceptance of $30 \%$ ) or among the $q-1$ values $\exp (2 \pi i n / q)$, where $n$ is an integer chosen uniformly among $1, \ldots, q-1)$.

\section{A. Results for $N=2$ \\ 1. Results along lines at fixed $J$}

To begin with, we investigate the behavior of the model along lines with fixed $J$. We present a detailed study at $J=0.8$ (we consider sizes $16 \leqslant L \leqslant 64$ ) and some additional results for $J=0.6$, which, as we shall discuss, is quite close to the MCP.

Since the value $J=0.8$ is larger than $J_{c}(\kappa=0)=$ $0.7102(1)$, we expect, by increasing $\kappa$, to intersect the OC-OD line; see Fig. 1. No other transition is expected if the MCP satisfies $J_{m}<0.8$ (we will verify below that $J_{m} \lesssim 0.56$ ). In Fig. 2 we show the specific heats $C_{g}$ and $C_{z}$ as a function of $\kappa$ at fixed $J=0.8$. We observe a peak for $\kappa \approx 0.47$, which confirms the presence of a phase transition. Quantities related to the observable $Q$ do not show any singular behavior (although they are expected to be nonanalytic at the transition), confirming that the transition belongs to the OC-OD line. The 


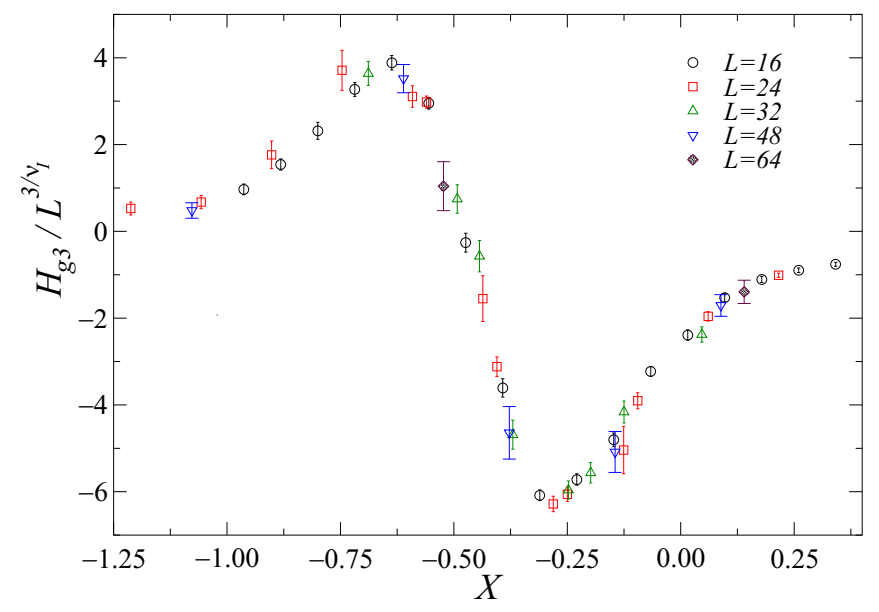

FIG. 3. Scaling plot of the cumulant $H_{g 3}$ as a function of $X=$ $\left(\kappa-\kappa_{c}\right) L^{1 / \nu_{I}}$, taking $v_{I}=0.629971$ and $\kappa_{c}=0.47131$. Results for $J=0.8$ and $N=2$.

Binder parameter $U$ and the correlation length $\xi$ do not vary significantly in the transition region. However, they vary with the size of the system: $U \approx 1.0011,1.0003,1.00006$ and $\xi \approx$ $28.7,81,230$ for $L=16,32$, and 64 . Note that $\xi$ increases faster than $L$ with the size of the box, as expected for an ordered phase-we expect that $\xi \sim L^{2}$ asymptotically. To characterize the critical behavior, as suggested in Ref. [48], we consider the scaling behavior of the third moment $H_{g 3}$, which is expected to scale as

$$
H_{g 3} \approx L^{3 / v}\left[f_{3}(X)+L^{-\omega} g_{3}(X)+L^{3-3 / v} g_{3 a}(\kappa)\right],
$$

where $X=\left(\kappa-\kappa_{c}\right) L^{1 / v}, \omega$ is the leading correction-toscaling exponent and the last term is due to the analytic background. For an Ising transition [78,79]

$$
v_{I}=0.629971(4), \quad \omega_{I}=0.832(6),
$$

and $3-3 / v_{I}=-1.76212(3)$. Corrections decay quite rapidly and the analytic background is negligible. Note that this is not the case of the specific heats that scale as

$$
C_{g, z} \approx L^{2 / \nu-3}\left[f_{C}(X)+L^{-\omega} g_{C}(X)+L^{3-2 / v} g_{C a}(\kappa)\right] .
$$

In this case, since $3-2 / v_{I} \approx-0.17475(2)$, the analytic term gives rise to quite slowly decaying scaling corrections.

To verify the Ising nature of the transition, we perform nonlinear fits to Eq. (22), approximating $f_{3}(X)$ with a polynomial of degree $n$ and neglecting all scaling corrections [we set $g_{3}(X)=g_{3 a}(\kappa)=0$ ]. If we take $n=14$, then we obtain $v=0.630(4)$ if we use all data and $v=0.623(10)$ if we only include data with $L \geqslant 24$. The results are clearly compatible with the Ising value $v_{I}$ reported in Eq. (23). To obtain a more precise estimate of $\kappa_{c}$ we have repeated the fits fixing $v$ to the Ising value reported above. A fit to all data gives

$$
\kappa_{c}=0.47131(2)
$$

which is in full agreement with the result obtained using only results with $L \geqslant 24, \kappa_{c}=0.47128(3)$. In Fig. 3 we report the scaling plot of $H_{g 3} L^{-3 / v}$ versus $X$, using the Ising value $v_{I}$ and

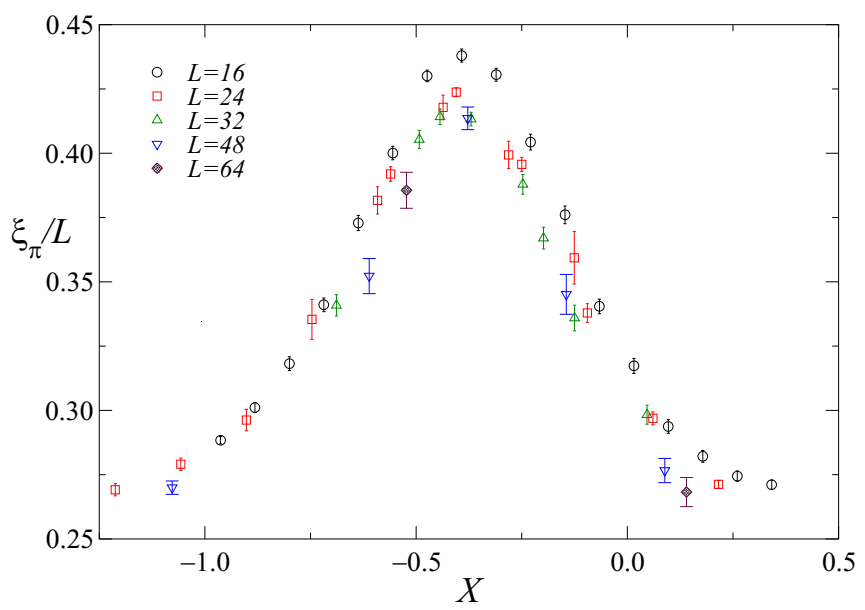

FIG. 4. Scaling plot of the ratio $\xi_{\pi} / L$ as a function of $X=(\kappa-$ $\left.\kappa_{c}\right) L^{1 / v_{I}}$, taking $v_{I}=0.629971$ and $\kappa_{c}=0.47131$. Results for $J=$ 0.8 and $N=2$.

the above-reported estimate of $\kappa_{c}$. We observe an excellent agreement.

To further confirm the presence of long-range correlations, we consider the correlation length $\xi_{\pi}$, obtained from the correlation function $G_{\pi}$, Eq. (13). In Fig. 4 we report $\xi_{\pi} / L$ as a function of $X=\left(\kappa-\kappa_{c}\right) L^{1 / \nu}$, using $v_{I}$ and the estimate of $\kappa_{c}$ reported in Eq. (25). We observe a reasonable good scaling, although not perfect, plausibly because of the corrections due to the analytic background. We also considered the correlation length $\xi_{F}$, which, however, is very small and consistent with zero within errors.

We have repeated the analysis for $J=0.6$, which is smaller than $J_{c}(\kappa=0)=0.7102(1)$ [57] and larger than $J_{c}(\kappa=\infty)=0.233965(2)$ [68]. By looking at Fig. 1, we see that, by increasing $J$ there are two possibilities: the line can intersect either one or two transition lines. The nature of the transition lines depends on the position of the MCP. If $J_{m}<J_{c}(\kappa=0)$ as in Fig. 1, then there are two possibilities: if $J>J_{m}$, by increasing $\kappa$, one would first cross the DC-OC line and then the OC-OD line; in the opposite case, $J<J_{m}$, one would observe only one transition on the DC-OD line. However, if $J_{m}>J_{c}(\kappa=0)$, then we should observe a single transition on the DC-OD line. As we discuss below, we find two transitions, a small- $\kappa$ Heisenberg one - therefore on the DC-OC line-and a large- $\kappa$ Ising one-therefore on the OC-OD line. We can thus conclude that

$$
J_{m}<0.6<J_{c}(\kappa=0),
$$

confirming the qualitative correctness of Fig. 1.

In Fig. 5 we report the specific heats as a function of $\kappa$. The plot of $C_{z}$ indicates the presence of two different transitions, one at $\kappa_{c} \approx 0.50$ and a second one at $\kappa_{c} \approx 0.54$. Note that $C_{g}$ is little sensitive to the first transition, which is clearly related to the condensation of the $Q$ field. The gauge degrees of freedom are only relevant for the largest- $\kappa$ transition.

To characterize the large- $\kappa$ transition, we consider the data corresponding to $\kappa>0.53$ and fit $H_{g 3}$ to $L^{3 / v} f_{3}(X)$ (we take a 14 th-order polynomial approximation). We find $v=$ 

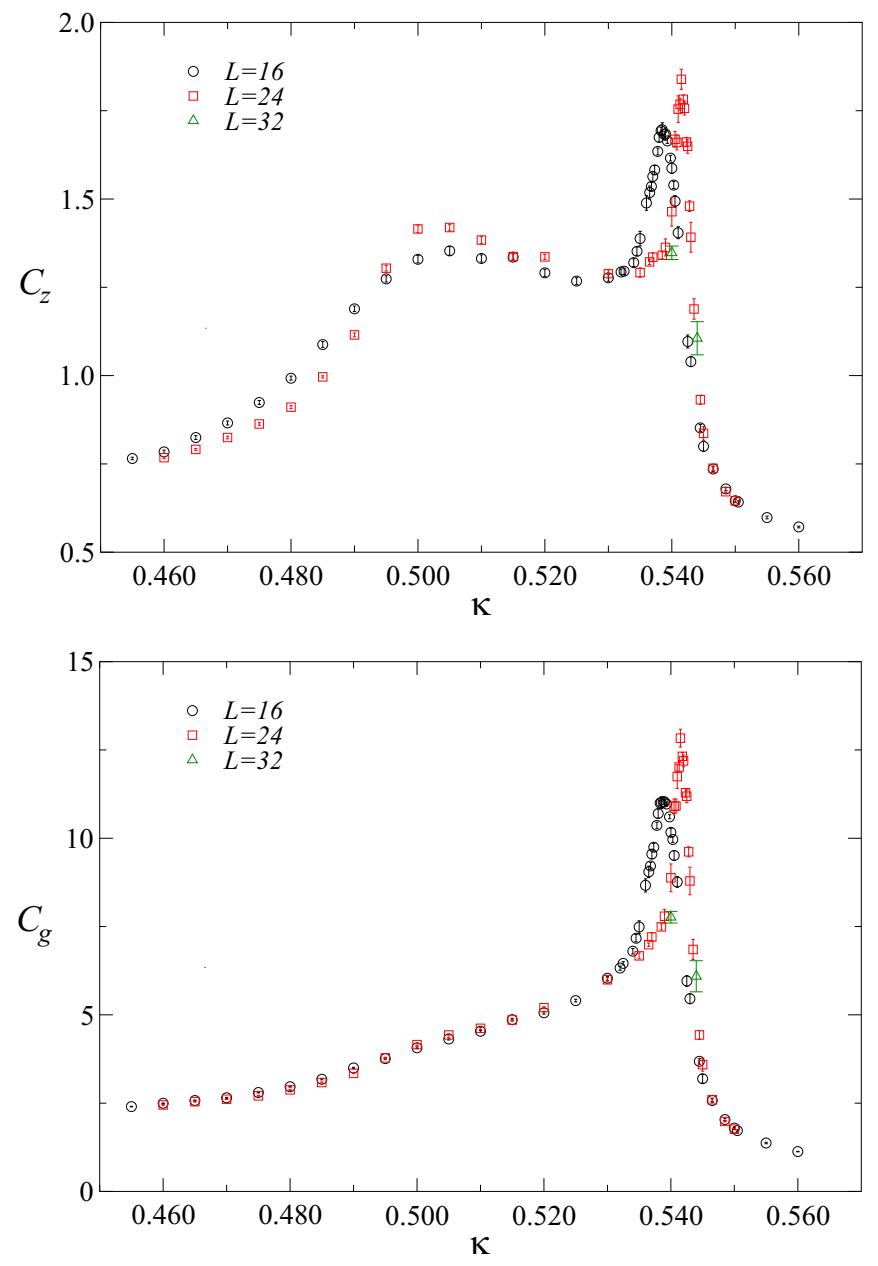

FIG. 5. Plot of the specific heats $C_{z}$ (top) and $C_{g}$ (bottom) as a function of $\kappa$ for $J=0.6$ and $N=2$.

$0.625(7)$, consistent with the Ising value Eq. (23). If we fix $v$ to the Ising value, we obtain $\kappa_{c}=0.54472(5)$. The error here only represents the statistical uncertainty of the fit. The systematic error due to the scaling corrections is probably larger. In Fig. 6 we report the corresponding scaling plot. The scaling is excellent. Comparing the estimates of $\kappa_{c}$ for $J=\infty$ $\left(\kappa_{c} \approx 0.381\right), J=0.8$ and $J=0.6$, we see that $\kappa_{c}$ decreases with increasing $J$, as sketched in Fig. 1. Moreover, we can derive a lower bound on the position of the MCP, $\kappa_{m} \gtrsim 0.55$.

To clarify the nature of the lower- $\kappa$ transition, we plot $U$ versus $R_{\xi}$, see Fig. 7 , considering only data with $\kappa<0.52$. Data fall onto a single curve, compatible with the universal curve computed for the Heisenberg universality class [80]. To determine the critical temperature we have performed fits of $U$ and $R_{\xi}$ to

$$
R=f(X), \quad X=\left(\kappa-\kappa_{c}\right) L^{1 / v},
$$

taking $v$ equal to the Heisenberg value [59] $v_{H}=$ 0.71164(10). Using a polynomial approximation for $f(X)$, we obtain $\kappa_{c}=0.4998(2)$ (fit of $U$ ) and $\kappa_{c}=0.4996$ (1) (fit of $\left.R_{\xi}\right)$. Scaling corrections are apparently of the order of the statistical errors. We take $\kappa_{c}=0.4997(3)$ as our final estimate.

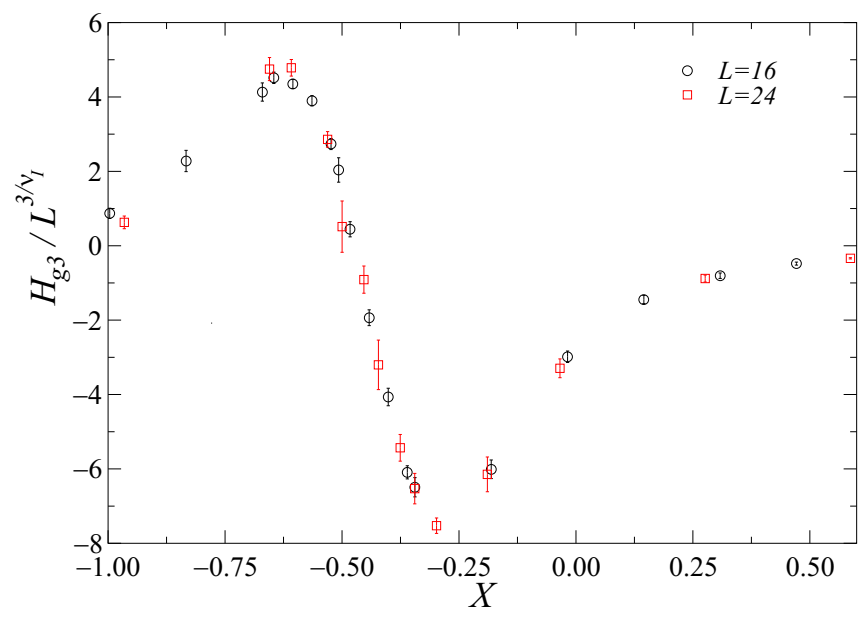

FIG. 6. Scaling plot of the cumulant $H_{g 3}$ as a function of $X=$ $\left(\kappa-\kappa_{c}\right) L^{1 / \nu_{I}}$, at the Ising transition taking $\nu_{I}=0.629971$ and $\kappa_{c}=$ 0.54472 . Results for $J=0.6$ and $N=2$. We only include data with $\kappa>0.53$.

\section{Results along lines at fixed $\kappa$}

We now study the behavior of the model by performing simulations at fixed $\kappa$. We perform simulations at $\kappa=0.4$, 0.55 , and 1 .

Since $\kappa_{c}(J=\infty)<0.4<\kappa_{m}$ (we estimated $\left.\kappa_{m} \gtrsim 0.55\right)$, Fig. 1 allows us to predict that, by increasing $J$ we should first cross the DC-OC line and, subsequently, the OC-OD line. We focus here on the low- $J$ transition. The estimates of $U$ and $R_{\xi}$ indicate that it occurs for $J \approx 0.65$. To verify that it belongs to the Heisenberg universality class, we plot $U$ versus $R_{\xi}$ and compare the results with the universal curve computed in the Heisenberg model, see Fig. 8. We observe an excellent agreement. We have also performed fits of $U$ and $R_{\xi}$ to Eq. (27), with $J$ replacing $\kappa$. We obtain $v=0.721(7)$ and 0.720 (3) from $U$ and $R_{\xi}$, respectively. We observe tiny deviations from the Heisenberg value [59] $v_{H}=0.71164(10)$,

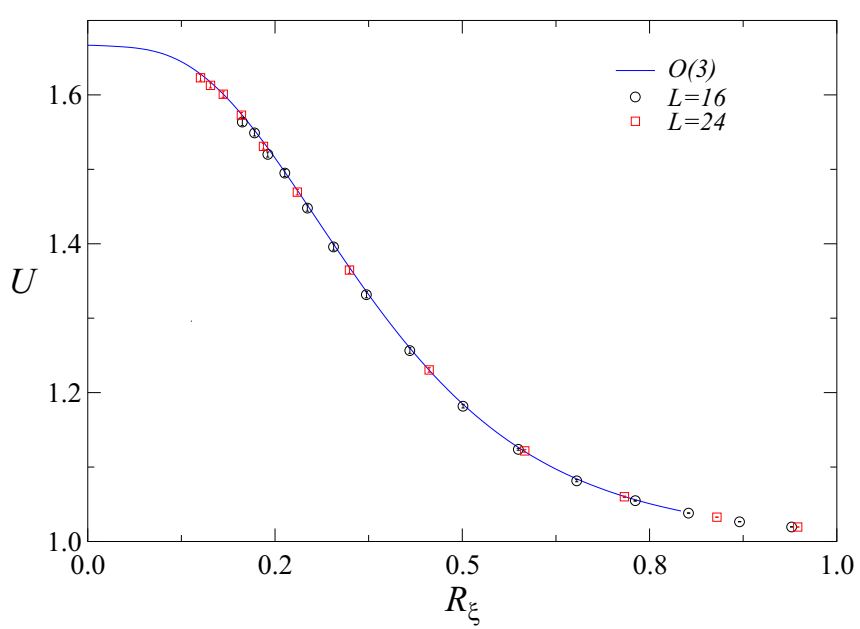

FIG. 7. Scaling plot of $U$ as a function of $R_{\xi}$, at the Heisenberg transition. Results for $J=0.6$ and $N=2$. We only include data with $\kappa<0.52$. The continuous line is the universal curve $U=F\left(R_{\xi}\right)$ in the standard Heisenberg model [80]. 


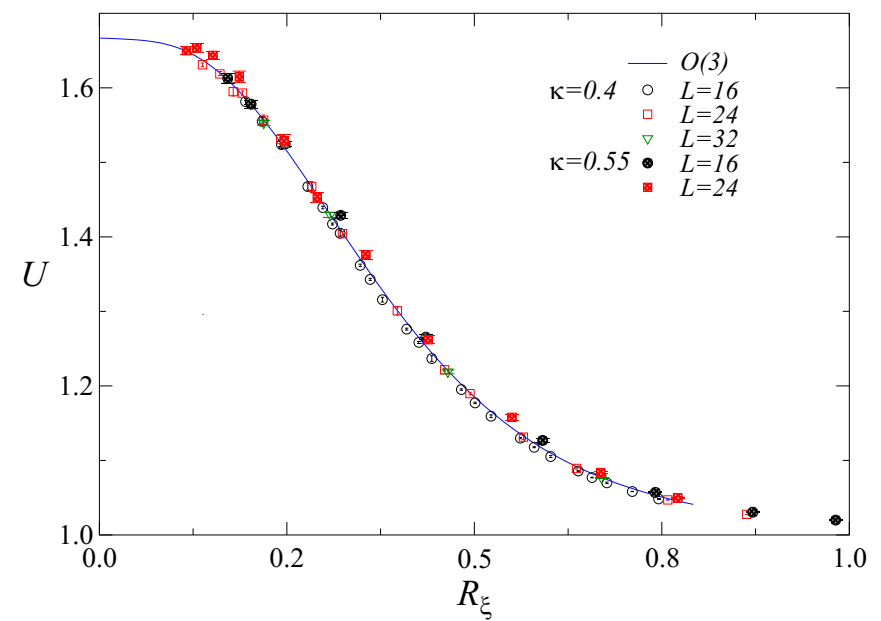

FIG. 8. Scaling plot of $U$ as a function of $R_{\xi}$. We report data for $N=2$, and $\kappa=0.4$ and $\kappa=0.55$. The continuous line is the universal curve $U=F\left(R_{\xi}\right)$ in the standard Heisenberg model [80].

which are most likely due to scaling corrections. If we fix $v=$ $v_{H}$, then we obtain $J_{c}=0.65001(4)$ and $J_{c}=0.649708(3)$ from the two fits. Statistical errors are clearly smaller than the systematic deviations due to the scaling corrections. A conservative estimate is $J_{c}=0.64985(20)$.

We have performed a second set of runs for $\kappa=0.55$. From the analysis of the data at fixed $J$ we already know that there is an Ising transition for $J \approx 0.6$. We wish now to identify the Heisenberg transition. We compute $U$ on lattices of size $L=16$ and 24 . We observe a rapid crossover from $5 / 3$ to 1 as $J$ varies from 0.54 and 0.58 , allowing us to identify the transition region. The results are plotted versus $R_{\xi}$ in Fig. 8. They are very close to the Heisenberg line, but not exactly on it on the scale of the figure: all of them are slightly above the scaling curve. We confirm therefore that the transition is a Heisenberg one, but observe that scaling corrections are now sizable, as expected since we are now close to the MCP. As before, we have performed fits of $U$ and $R_{\xi}$ to Eq. (27). We obtain estimates of $v$ that are consistent with the Heisenberg value: $v=0.70(1)$ and 0.72(3) from the analysis of $R_{\xi}$ and $U$. If $v$ is fixed to the Heisenberg value, then we estimate $J_{c}=0.56419(7)$ and $J_{c}=0.56422(12)$, respectively. Our final estimate is $J_{c}=0.5642(2)$.

Finally, we perform runs for $\kappa=1$, which allows us to study the behavior along the DC-OD line that connects the MCP with the $\mathrm{O}(4)$ point at $\kappa=\infty$. In Fig. 9 we report the specific heats $C_{z}$ and $C_{g}$, that signal a transition for $J \approx 0.355$, which is clearly not a Heisenberg one: in the latter case, since [56] $\alpha<0$, the specific heat does not increase with the size $L$. In Fig. 10 we report $U$ versus $R_{\xi}$. The data do not scale and, moreover, $U$ has a maximum whose height apparently increases as $L$ increases. This is the signature of a first-order transition, with a critical temperature $J_{c}=0.354(1)$. We also report $\xi_{\pi} / L$ versus $R_{\xi}$, see Fig. 11 . Also the energy-related correlation length increases with $L$, but with a finite-size behavior that appears to be unrelated to that of $\xi$. Finally, in Fig. 12 we report $\xi_{F}$. At all transition points we have considered above, $\xi_{F} \approx 0$. At this transition $\xi_{F}$ is sizable, but significantly smaller than $\xi$ and $\xi_{\pi}$. Moreover, it does not
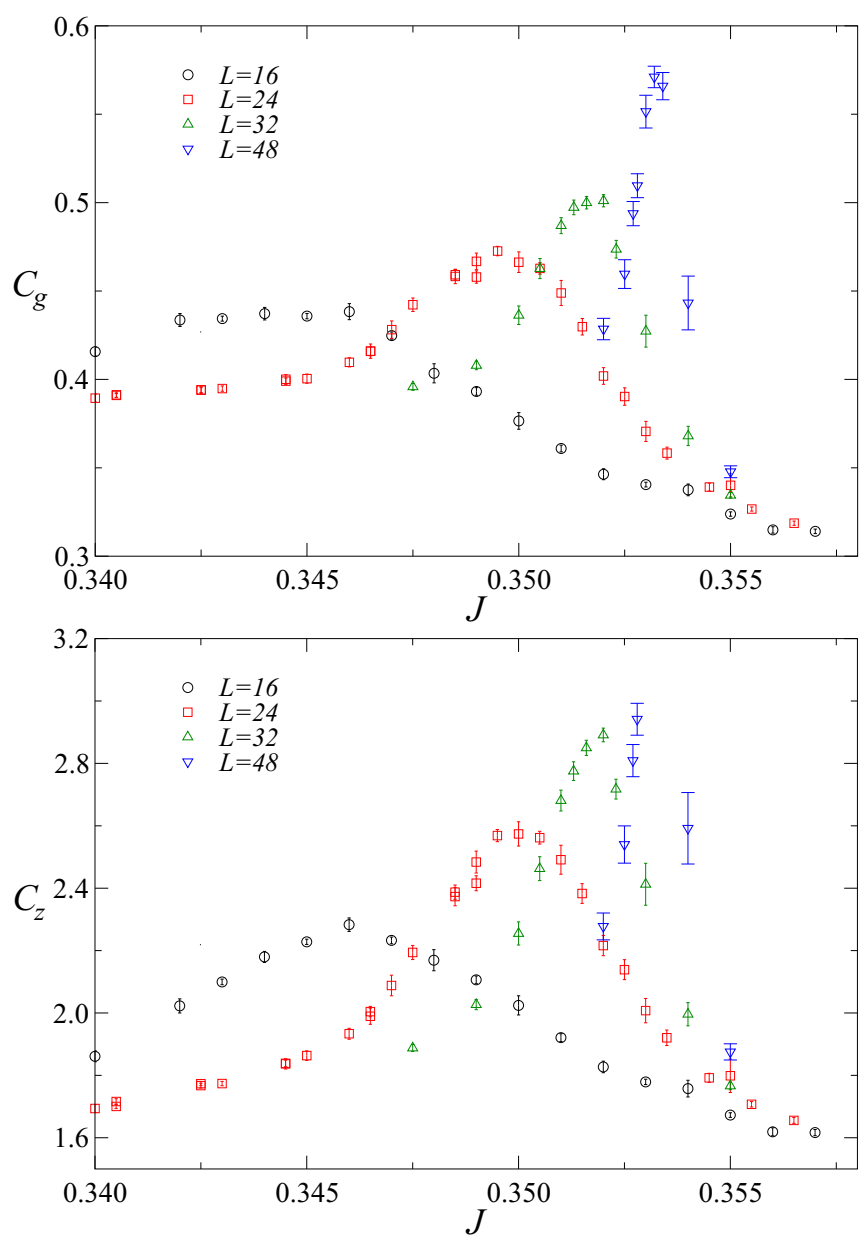

FIG. 9. Plot of $C_{g}$ (top) and of $C_{z}$ (bottom) as a function of $J$. Results for $\kappa=1$ and $N=2$.

increase with $L$, indicating that the modes encoded by this correlation function are not those that drive the transition.

\section{B. Results for $N=25$}

We now consider the $\mathrm{CP}^{24}$ model and perform an analysis analogous to the one presented for $N=2$. According to Ref. [58], the compact $\mathrm{CP}^{19}$ model has a critical transition at $J_{c}=0.353(2)$. Since the transition point in the $\mathrm{CP}^{N-1}$ model decreases with increasing $N$, the $\mathrm{CP}^{24}$ model should have a transition at $J_{c} \lesssim 0.35$. Therefore, to investigate the behavior of the model along the OC-OD critical line that connects the MCP with the Ising transition at $J=\infty$, we have performed a run at fixed $J=0.4$. We find that the specific heat $C_{g}$ has a clear maximum that increases with $L$ for $\kappa \approx 0.40$, that we identify as the critical transition. We analyze the behavior of the cumulant $H_{g 3}$ close to the transition, fitting the data to $L^{3 / v_{I}} f(X)$, where $X=\left(\kappa-\kappa_{c}\right) L^{1 / v_{I}}$, using $v_{I}=0.629971(4)$ [78]. Data scale nicely, see Fig. 13, confirming the Ising nature of the transition line. We estimate $\kappa_{c}=0.40200(5)$, where the error takes into account only the statistical fluctuations of the fit - the systematic error due to the scaling correction is probably larger.

We now focus on the DC-OC transition line that connects the $\mathrm{CP}^{24}$ transition point with the MCP. We perform runs 


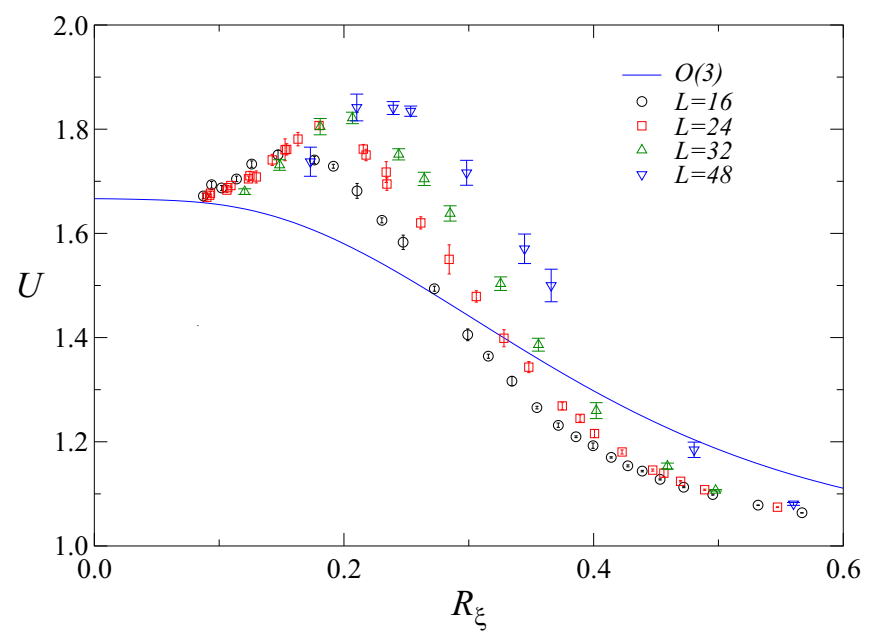

FIG. 10. Scaling plot of $U$ as a function of $R_{\xi}$. Results for $\kappa=1$ and $N=2$. The continuous line is the universal curve $U=F\left(R_{\xi}\right)$ in the standard Heisenberg model [80].

at $\kappa=0.2$ on lattices $L=16$, observing strong hysteresis effects for $J \approx 0.339$. The transitions on the DC-OC line are therefore of first order, as the $\mathrm{CP}^{24}$ transition for $\kappa=0$.

Finally, we focus on the DC-OD line connecting the MCP with the $\mathrm{O}(50)$ transition point for $\kappa=\infty$. We perform runs for $\kappa=1$, which should be well above the MCP. On the basis of our results for the Ising line-the results at fixed $J=0.4-$ we expect the MCP to have $\kappa_{m} \approx 0.4-0.5$. In Fig. 14 we show the specific heats as a function of $J$. The quantity $C_{z}$ has a well-defined maximum for $J \approx 0.29$, which does not increase with the size. This is a clear sign of a continuous transition with $\alpha<0$. The specific heat $C_{g}$ instead seems to be loosely coupled with the transition: it only shows short plateaus for the values of $J$ at which $C_{z}$ has a maximum. The transition is clearly related to the condensation of the degrees of freedom associated with the field $z$. In Fig. 15 we report $U$ versus $R_{\xi}$. Data scale nicely, confirming the continuous nature of the transition.

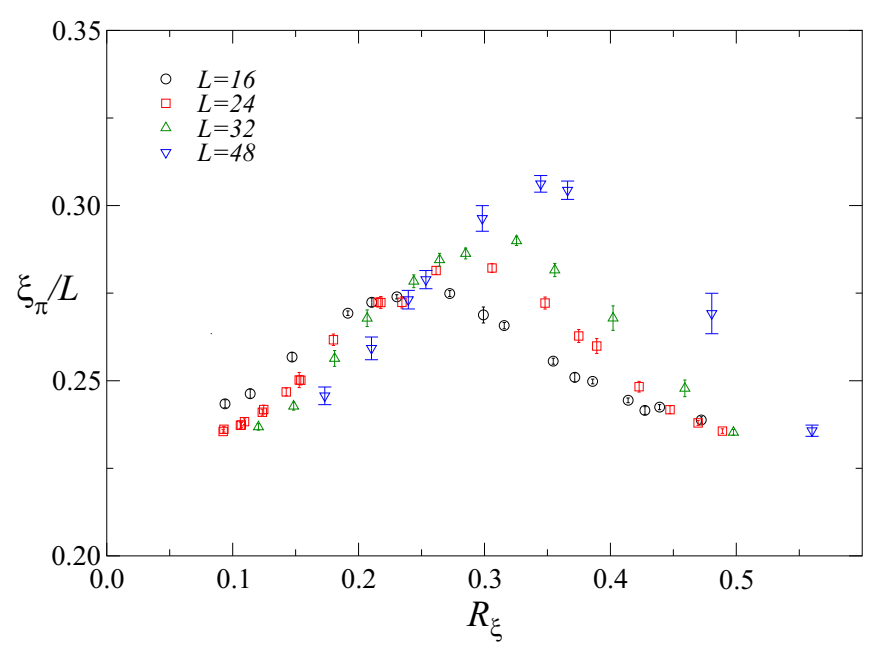

FIG. 11. Scaling plot of $\xi_{\pi} / L$ as a function of $R_{\xi}$. Results for $\kappa=1$ and $N=2$.

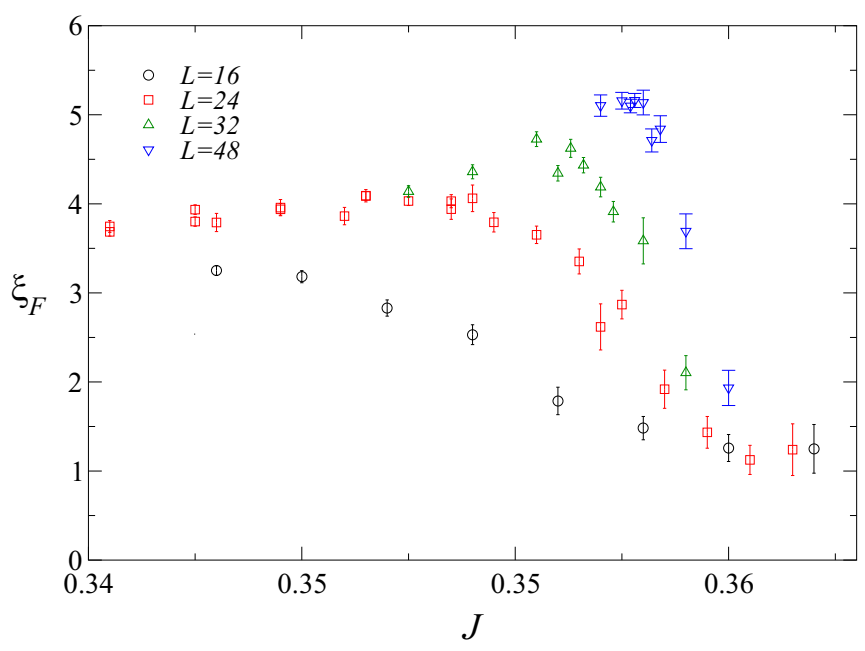

FIG. 12. Scaling plot of $\xi_{F}$ as a function of $J$. Results for $\kappa=1$ and $N=2$.

We now proceed to the computation of the critical indices. To estimate the correlation-length exponent $v$, we first perform fits to Eq. (27), where we use a polynomial approximation for $f(X)$. We obtain $v=0.788(2)$ and $v=0.796(4)$ from the analysis of $R_{\xi}$ and $U$, respectively. However, the $\chi^{2}$ (weighted sum of the fit residuals) for the fit of $R_{\xi}$ is large: $\chi^{2} / \mathrm{DOF} \approx 2.5$, where DOF is the number of degrees of freedom of the fit. This may indicate that there are sizable scaling corrections. Therefore, we performed a second set of fits to

$$
R=f(X)+L^{-\omega} g(X),
$$

which includes the leading scaling corrections. The $\chi^{2}$ of the fit is little sensitive to $\omega$ in the range $\omega \gtrsim 0.5$. Varying $\omega$ in this range we can estimate

$$
v=0.815(15), \quad J_{c}=0.29333(3) .
$$

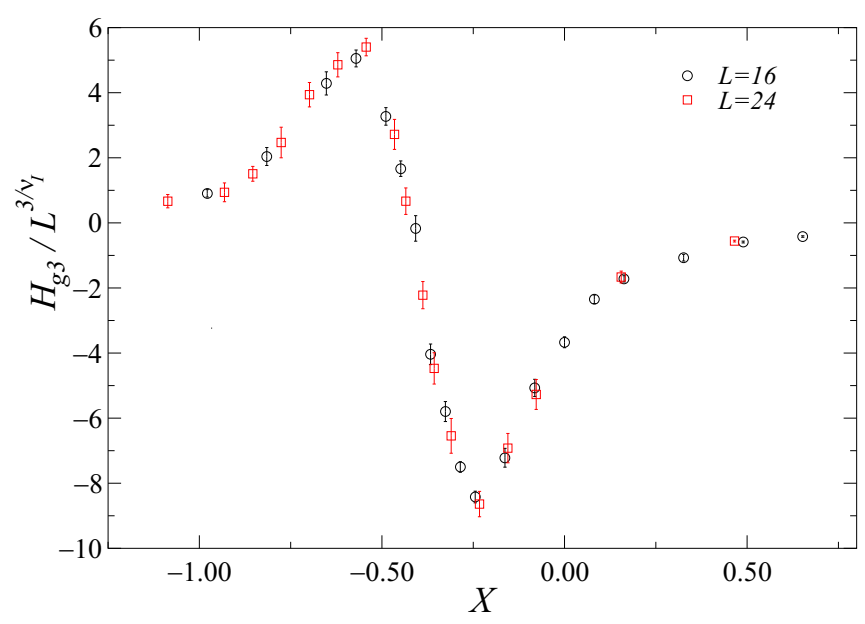

FIG. 13. Scaling plot of the cumulant $H_{g 3}$ as a function of $X=$ $\left(\kappa-\kappa_{c}\right) L^{1 / v_{I}}$, for $v_{I}=0.629971$ and $\kappa_{c}=0.40200$. Results for $J=$ 0.4 and $N=25$. 

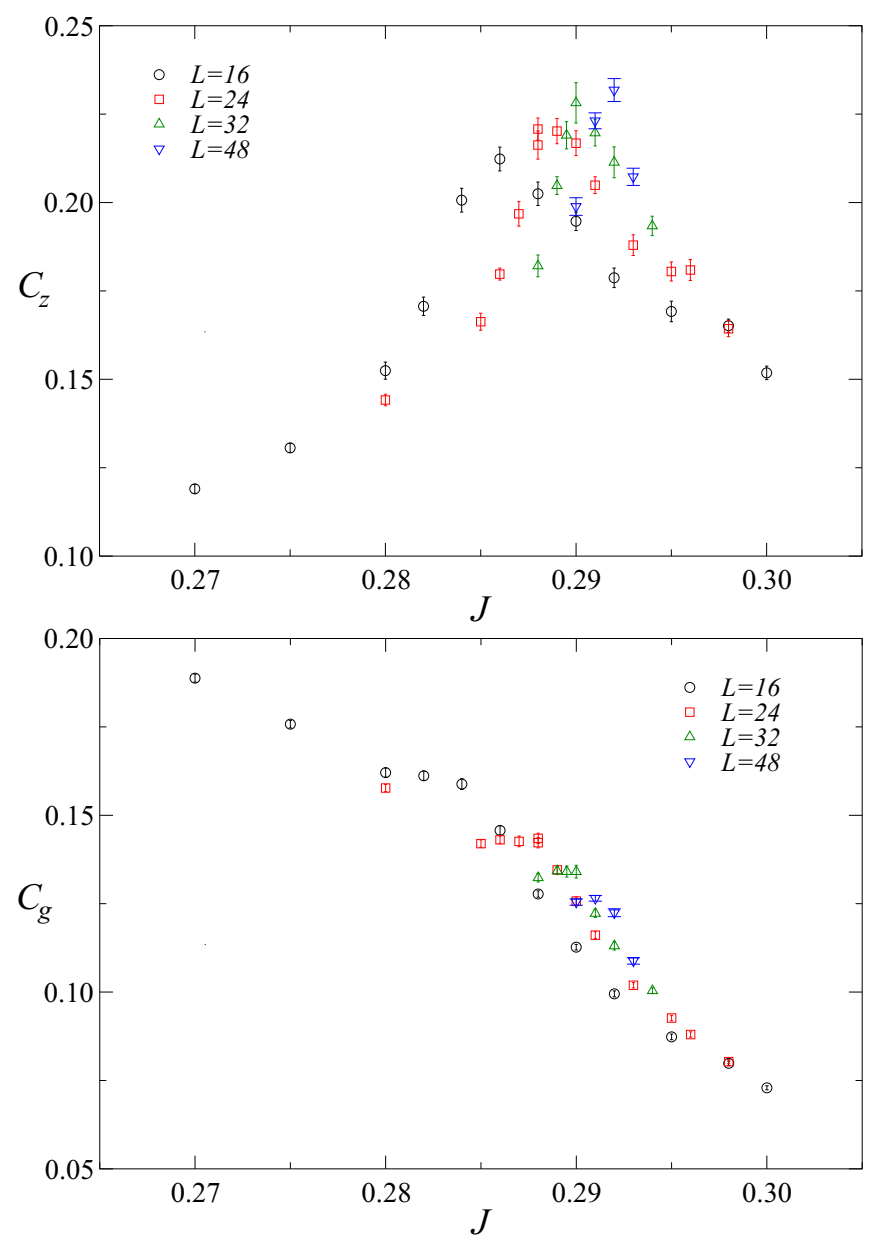

FIG. 14. Plot of the specific heats $C_{z}$ (top) and $C_{g}$ (bottom) as a function of $J$ for $\kappa=1$ and $N=25$.

The corresponding scaling plots of $U$ and $R_{\xi}$ are reported in Fig. 16. We finally estimate the exponent $\eta_{q}$ defined by the size dependence of the susceptibility $\chi$ at the critical point:

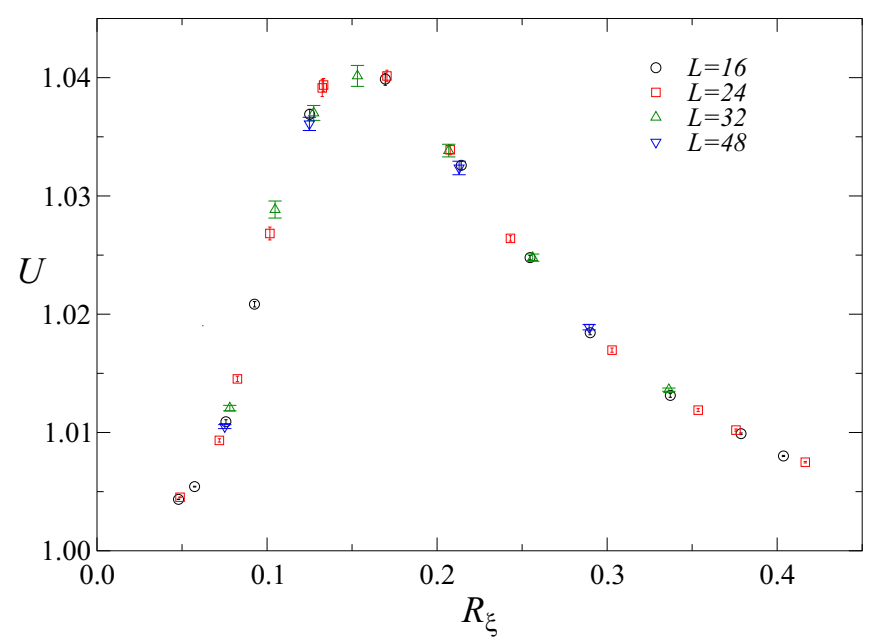

FIG. 15. Scaling plot of $U$ as a function of $R_{\xi}$. Results for $\kappa=1$ and $N=25$.
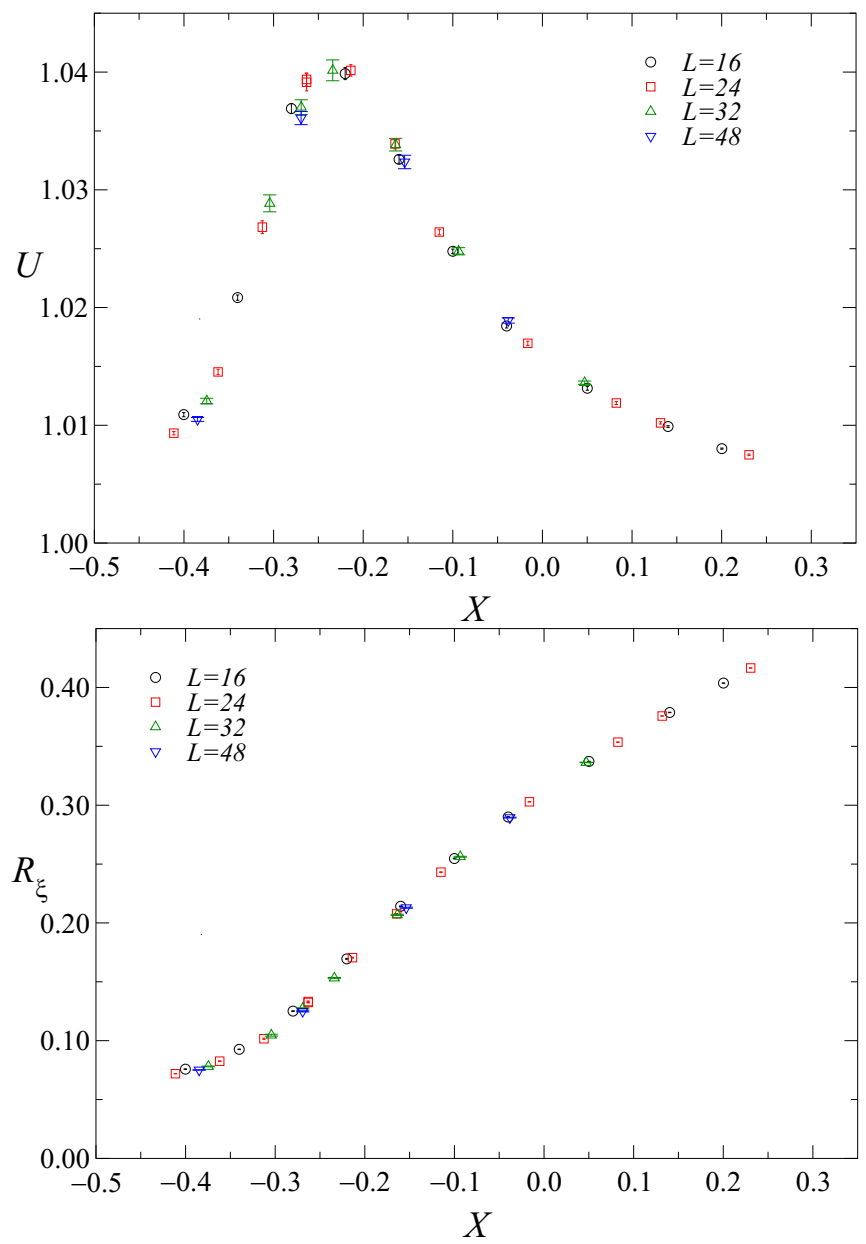

FIG. 16. Scaling plot of $U$ (top) and $R_{\xi}$ versus $X=\left(J-J_{c}\right) L^{1 / v}$, for $\nu=0.815$ and $J_{c}=0.29333$. Results for $\kappa=1$ and $N=25$.

$\chi \sim L^{2-\eta_{q}}$. We perform fits to [45]

$$
\chi=L^{2-\eta_{q}}\left[f_{\chi}\left(R_{\xi}\right)+L^{-\omega} g_{\chi}\left(R_{\xi}\right)\right] .
$$

Correspondingly, we obtain the estimate

$$
\eta_{q}=0.88(2) \text {. }
$$

The fit is also sensitive to $\omega$ and gives $\omega=1.1(2)$. This estimate is quite reasonable, since we expect $\omega \rightarrow 1$ in the limit $N \rightarrow \infty$. The quality of the fit is excellent, see Fig. 17.

\section{SUPPRESSING THE MONOPOLES IN THE HIGHER-CHARGE $\mathrm{CP}^{N-1}$ MODEL}

In the previous sections we have discussed the phase diagram of the AH compact model with $q=2$. As it has been discussed at length in the literature [12-14,23-27], the critical behavior is supposed to depend on the topology of the gauge fields and in particular on the presence/absence of monopoles. In particular, it was shown in our previous work [27] on the $q=1$ monopole-free $\mathrm{CP}^{N-1}$ model, that monopole suppression leads to a different critical behavior. No Heisenberg transition is observed for $N=2$, while for $N=25$ a novel critical behavior appears, whose interpretation within the field-theory framework is still obscure [45]. 


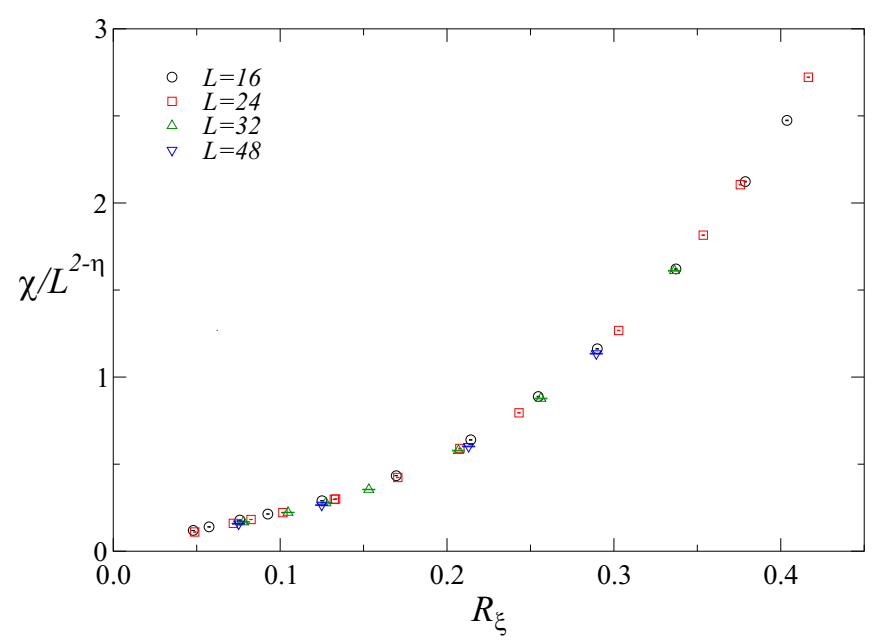

FIG. 17. Scaling plot of $L^{-2+\eta_{q}} \chi$ versus $R_{\xi}$ for $\eta_{q}=0.88$. Results for $\kappa=1$ and $N=25$.

We wish now to investigate the role that monopoles play in higher-charge systems. As in Ref. [27], we use the De Grand-Toussaint [81] monopole definition and consider the model in which monopoles are absent. We shall focus on the model with $\kappa=0$, that will be referred to as the monopolefree $\mathrm{CP}^{N-1}\left(\mathrm{MFCP}^{N-1}\right)$ model. As we shall discuss below, this will be enough to conjecture a plausible phase diagram for any $\kappa>0$.

For $\kappa=0$, in the absence of the monopole constraint, the model with charge- $q$ fields is obviously equivalent to the usual one. However, the introduction of the monopole-suppression constraint which is applied to the $\lambda$ fields breaks the equivalence and thus a different behavior is possible. We have performed numerical simulations, studying two cases, $q=2$ and $q=3$.

Let us first consider the $\mathrm{MFCP}^{1}$. We have performed simulations on systems with $L=16,24,32$. In Fig. 18 we show our numerical estimates of $U$ versus $R_{\xi}$. Data scale nicely, clearly indicating that scaling corrections are small. Moreover,

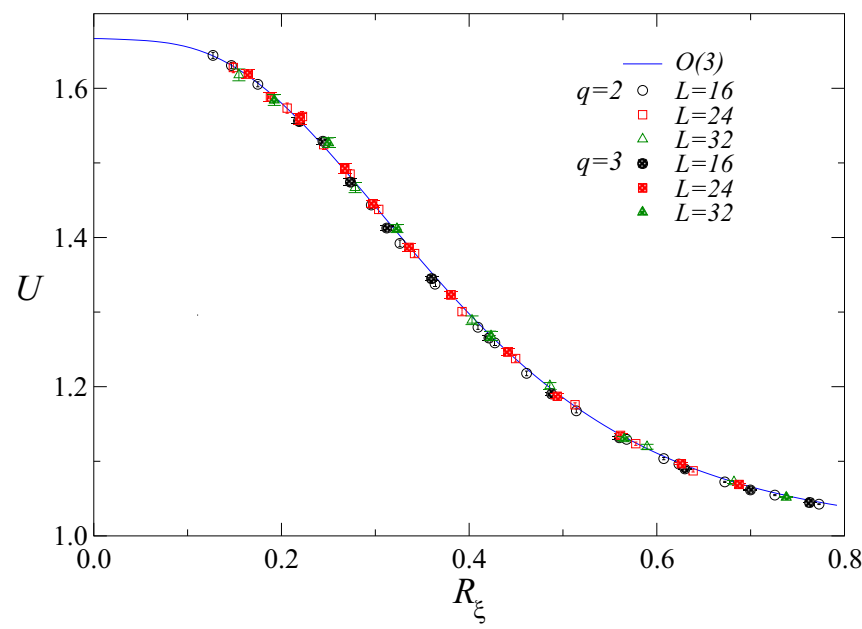

FIG. 18. Plot of the Binder parameter $U$ versus $R_{\xi}$ for $q=2$ and $q=3$ for the $\mathrm{MFCP}^{1}$ model. The continuous line is the universal curve $U=F\left(R_{\xi}\right)$ in the standard Heisenberg model [80]. data fall on top of the $\mathrm{O}(3)$ scaling curve, allowing us to infer that the transition, for both values of $q$, belongs to the $\mathrm{O}(3)$ universality class, as in the standard $\mathrm{CP}^{1}$ model without monopole suppression. Apparently the monopole constraint, which is crucial in determining the critical behavior for $q=1$, plays no role for $q \geqslant 2$. We also performed fits of the data to

$$
R(\beta, L)=f_{R}\left[\left(J-J_{c}\right) L^{1 / \nu}\right]
$$

taking $R=R_{\xi}$ and $U$, and using a polynomial approximation for $f_{R}(x)$. We find that the estimates of $v$ are consistent with the Heisenberg value, $v_{H} \approx 0.711$. Fixing $v$ to the Heisenberg value [59] $v_{H}=0.71164(10)$, we can estimate the critical temperature: $J_{c}=0.7074(3)$ for $q=2$ and $J_{c}=0.6980(6)$ for $q=3$. These two estimates are very close to the transition temperature for the standard $\mathrm{CP}^{1}$ model, $J_{c}=0.7102(1)$, while they differ significantly from the $q=1 \mathrm{MFCP}^{1}$ value, $J_{c}=0.4605(3)$. It is quite clear that the monopole constraint is not effective for $q \geqslant 2$. To verify the irrelevance of monopole suppression we have also performed simulations with $q=2$ and larger values of $N$. For $N=10,20$ we observe strong first-order transitions, that are completely analogous to those observed in the standard $\mathrm{CP}^{N-1}$ model. Apparently, monopole suppression is not effective for any value of $N$.

The results obtained for $\kappa=0$ allow us to conjecture that monopole suppression does not play any role for any $\kappa>0$. Indeed, the introduction of the interaction term $H_{g}$ defined in Eq. (3) leads to a reduction of the number of monopoles as $\kappa$ increases. Thus, we expect the role of monopole suppression to be maximal for $\kappa=0$. Therefore, the irrelevance for $\kappa=0$ leads us to predict that they irrelevant for any $\kappa>0$.

\section{CONCLUSIONS}

We have considered 3D lattice $\mathrm{AH}$ models with compact gauge fields and multicomponent complex scalar fields with integer charge $q$. We have discussed the dependence on the charge $q$ of the phase diagram and the nature of the phase transitions.

The resulting phase diagram for $q=2$ - but we expect a qualititively similar diagram for any $q \geqslant 2$-and unit-length scalar fields (London limit) is sketched in Fig. 1. There are three phases, separated by three different transition lines meeting at a MCP. There is one phase (DC phase) in which the scalar fields are disordered-the $\mathrm{SU}(N)$ global symmetry is unbroken - and single-charge external particles are confined-Wilson loops obey the area law for large sizes. In the other two phases (OC and OD) the $\mathrm{SU}(N)$ symmetry is broken. They differ in the behavior of single-charge external particles: they are confined for small $\kappa$ (OC phase), deconfined for large $\kappa$ (OD phase).

The nature of the transition lines depends on the number of components. For $N=2$ the transitions along the DC-OC line are continuous, belonging to the $\mathrm{O}(3)$ vector universality classes. Along this line the scalar-field degrees of freedom order, the $\mathrm{SU}(N)$ symmetry breaks, while gauge-field modes play no role. The transitions along the OC-OD line are continuous and belong to the Ising universality class. The transitions are driven by the gauge degrees of freedom, while the scalar field plays no role. Finally, first-order transitions 
are observed along the DC-OD line, ending to the $\mathrm{O}(4)$ critical point for $\kappa \rightarrow \infty$. The phase diagram substantially differs from that observed for the compact AH model with single-charge scalar fields [44], whose phase diagram has two phases, differing only in the behavior of the gauge-invariant scalar-field correlations; they are separated by a line of continuous transitions belonging to the $\mathrm{O}(3)$ vector universality class [44], where the gauge correlations do not play any role.

To characterize the large- $N$ behavior of the model, we have also reported a numerical study for $N=25$. The transitions along the OC-OD line are continuous and belong to the Ising universality class, like the case $N=2$. The behavior along the DC-OC and OC-OD line instead differs. Indeed, the DC-OC transitions are of first order, while the DC-OD transitions are continuous. We have determined the correlation-length exponent $v$ and the exponent $\eta_{q}$ that characterizes the critical behavior of the order parameter $Q_{x}$ defined in Eq. (6), on the DC-OD line. We find $v=0.815(15)$ and $\eta_{q}=0.88(2)$. Interestingly, they are in quantitative agreement with the exponents obtained along the Coulomb-Higgs transition line in the $\mathrm{AH}$ model with noncompact gauge fields [45]: $v=0.802(8)$ and $\eta_{q}=0.883$ (7). In Ref. [45] we conjectured that the Coulomb-
Higgs transitions in the noncompact $\mathrm{AH}$ model belong to the universality class associated with the stable fixed point of the multicomponent AH field theory [1,72,73], cf. Eq. (20). Indeed, the numerical results were in excellent agreement with the $1 / N$ predictions computed in the continuum AH model [1,82]: $v \approx 0.805$ and $\eta_{q} \approx 0.870$. This comparison leads us to conjecture that also the critical behavior of the large- $N$ continuous DC-OC transitions belongs to the universality class associated with the stable $3 \mathrm{D}$ fixed point of the $\mathrm{AH}$ field theory.

The analogies between the phase diagram of compact $\mathrm{AH}$ models with doubly charged multicomponent scalar fields and that of the noncompact $\mathrm{AH}$ models [45], and, in particular, the correspondence of the DC-OD critical behavior with the AH field theory, may appear quite unexpected, and certainly deserves further investigation. We finally note that the noncompact formulation of the AH theory is recovered in the $q \rightarrow \infty$ limit of the compact formulation, with an appropriate rescaling of the gauge couplings, $\kappa_{\text {nco }}=\kappa / q^{2}\left(\kappa_{\text {nco }}\right.$ in the inverse gauge coupling in the noncompact model). Thus, it is tempting to conjecture that the phase diagram and, in particular, the nature of the transitions along the DC-OD line, is the same for any $q \geqslant 2$.
[1] B. I. Halperin, T. C. Lubensky, and S. K. Ma, First-Order Phase Transitions in Superconductors and Smectic-A Liquid Crystals, Phys. Rev. Lett. 32, 292 (1974).

[2] C. Dasgupta and B. I. Halperin, Phase Transitions in a Lattice Model of Superconductivity, Phys. Rev. Lett 47, 1556 (1981).

[3] R. Folk and Y. Holovatch, On the critical fluctuations in superconductors, J. Phys. A 29, 3409 (1996).

[4] S. Mo, J. Hove, and A. Sudbø, Order of the metal-tosuperconductor transition, Phys. Rev. B 65, 104501 (2002).

[5] T. Neuhaus, A. Rajantie, and K. Rummukainen, Numerical study of duality and universality in a frozen superconductor, Phys. Rev. B 67, 014525 (2003).

[6] N. Read and S. Sachdev, Spin-Peierls, valence-bond solid, and Néel ground states of low-dimensional quantum antiferromagnets, Phys. Rev. B 42, 4568 (1990).

[7] S. Takashima, I. Ichinose, and T. Matsui, $\mathrm{CP}^{1}+\mathrm{U}(1)$ lattice gauge theory in three dimensions: Phase structure, spins, gauge bosons, and instantons, Phys. Rev. B 72, 075112 (2005).

[8] S. Takashima, I. Ichinose, and T. Matsui, Deconfinement of spinons on critical points: Multiflavor $\mathrm{CP}^{1}+\mathrm{U}(1)$ lattice gauge theory in three dimension, Phys. Rev. B 73, 075119 (2006).

[9] R. K. Kaul, Quantum phase transitions in bilayer $\mathrm{SU}(N)$ antiferromagnets, Phys. Rev. B 85, 180411(R) (2012).

[10] R. K. Kaul and A. W. Sandvik, Lattice Model for the SU(N) Néel to Valence-Bond Solid Quantum Phase Transition at Large N, Phys. Rev. Lett. 108, 137201 (2012).

[11] M. S. Block, R. G. Melko, and R. K. Kaul, Fate of $\mathrm{CP}^{N-1}$ Fixed Point with $q$ Monopoles, Phys. Rev. Lett. 111, 137202 (2013).

[12] A. Nahum, J. T. Chalker, P. Serna, M. Ortuño, and A. M. Somoza, Deconfined Quantum Criticality, Scaling Violations, and Classical Loop Models, Phys. Rev. X 5, 041048 (2015).

[13] C. Wang, A. Nahum, M. A. Metliski, C. Xu, and T. Senthil, Deconfined Quantum Critical Points: Symmetries and Dualities, Phys. Rev. X 7, 031051 (2017).
[14] T. Senthil, L. Balents, S. Sachdev, A. Vishwanath, and M. P. A. Fisher, Quantum criticality beyond the Landau-GinzburgWilson paradigm, Phys. Rev. B 70, 144407 (2004).

[15] A. W. Sandvik, Evidence for Deconfined Quantum Criticality in a Two-Dimensional Heisenberg Model with Four-Spin Interactions, Phys. Rev. Lett. 98, 227202 (2007).

[16] R. G. Melko and R. K. Kaul, Scaling in the Fan of an Unconventional Quantum Critical Point, Phys. Rev. Lett. 100, 017203 (2008).

[17] F.-J. Jiang, M. Nyfeler, S. Chandrasekharan, and U.-J. Wiese, From an antiferromagnet to a valence bond solid: Evidence for a first-order phase transition, J. Stat. Mech. (2008) P02009.

[18] A. W. Sandvik, Continuous Quantum Phase Transition Between an Antiferromagnet and a Valence-Bond Solid in Two Dimensions: Evidence for Logarithmic Corrections to Scaling, Phys. Rev. Lett. 104, 177201 (2010).

[19] K. Harada, T. Suzuki, T. Okubo, H. Matsuo, J. Lou, H. Watanabe, S. Todo, and N. Kawashima, Possibility of deconfined criticality in $\mathrm{SU}(N)$ Heisenberg models at small $N$, Phys. Rev. B 88, 220408(R) (2013).

[20] K. Chen, Y. Huang, Y. Deng, A. B. Kuklov, N. V. Prokof'ev, and B. V. Svistunov, Deconfined Criticality Flow in the Heisenberg Model with Ring-Exchange Interactions, Phys. Rev. Lett. 110, 185701 (2013).

[21] S. Pujari, K. Damle, and F. Alet, Nèel-State to ValenceBond-Solid Transition on the Honeycomb Lattice: Evidence for Deconfined Criticality, Phys. Rev. Lett. 111, 087203 (2013).

[22] H. Shao, W. Guo, and A. W. Sandvik, Quantum criticality with two length scales, Science 352, 213 (2016).

[23] G. Murthy and S. Sachdev, Actions of hedgehogs instantons in the disordered phase of $2+1$ dimensional $\mathrm{CP}^{N-1}$ model, Nucl. Phys. B 344, 557 (1990). 
[24] O. I. Motrunich and A. Vishwanath, Emergent photons and transitions in the $\mathrm{O}(3) \sigma$-model with hedgehog suppression, Phys. Rev. B 70, 075104 (2004).

[25] A. Nahum, J. T. Chalker, P. Serna, M. Ortuño, and A. M. Somoza, 3D Loop Models and the $\mathrm{CP}^{N-1}$ Sigma Model, Phys. Rev. Lett. 107, 110601 (2011).

[26] A. Nahum, J. T. Chalker, P. Serna, M. Ortuño, and A. M. Somoza, Phase transitions in three-dimensional loop models and the $\mathrm{CP}^{N-1} \sigma$ model, Phys. Rev. B 88, 134411 (2013).

[27] A. Pelissetto and E. Vicari, Three-dimensional monopole-free $\mathrm{CP}^{N-1}$ models, Phys. Rev. E 101, 062136 (2020).

[28] A. B. Kuklov, N. V. Prokof'ev, B. V. Svistunov, and M. Troyer, Deconfined criticality, runaway flow in the two-component scalar electrodynamics and weak first-order superfluid-solid transitions, Ann. Phys. 321, 1602 (2006).

[29] O. I. Motrunich and A. Vishwanath, Comparative study of Higgs transition in one-component and two-component lattice superconductor models, arXiv:0805.1494.

[30] A. B. Kuklov, M. Matsumoto, N. V. Prokof'ev, B. V. Svistunov, and M. Troyer, Deconfined Criticality: Generic First-Order Transition in the SU(2) Symmetry Case, Phys. Rev. Lett. 101, 050405 (2008).

[31] A. B. Kuklov, M. Matsumoto, N. V. Prokof'ev, B. V. Svistunov, and M. Troyer, Comment on "Comparative study of Higgs transition in one-component and two-component lattice superconductor models", arXiv:0805.2578.

[32] D. Charrier, F. Alet, and P. Pujol, Gauge Theory Picture of an Ordering Transition in a Dimer Model, Phys. Rev. Lett. 101, 167205 (2008).

[33] J. Lou, A. W. Sandvik, and N. Kawashima, Antiferromagnetic to valence-bond-solid transitions in two-dimensional SU(N) Heisenberg models with multispin interactions, Phys. Rev. B 80, 180414(R) (2009).

[34] G. Chen, J. Gukelberger, S. Trebst, F. Alet, and L. Balents, Coulomb gas transitions in three-dimensional classical dimer models, Phys. Rev. B 80, 045112 (2009).

[35] D. Charrier and F. Alet, Phase diagram of an extended classical dimer model, Phys. Rev. B 82, 014429 (2010).

[36] A. Banerjee, K. Damle, and F. Alet, Impurity spin texture at a deconfined quantum critical point, Phys. Rev. B 82, 155139 (2010).

[37] E. V. Herland, T. A. Bojesen, E. Babaev, and A. Sudbø, Phase structure and phase transitions in a three-dimensional SU(2) superconductor, Phys. Rev. B 87, 134503 (2013).

[38] L. Bartosch, Corrections to scaling in the critical theory of deconfined criticality, Phys. Rev. B 88, 195140 (2013).

[39] T. A. Bojesen and A. Sudbø, Berry phases, current lattices, and suppression of phase transitions in a lattice gauge theory of quantum antiferromagnets, Phys. Rev. B 88, 094412 (2013).

[40] A. Nahum, P. Serna, J. T. Chalker, M. Ortuño, and A. M. Somoza, Emergent $\mathrm{SO}(5)$ Symmetry at the Néel to Valence-Bond-Solid Transition, Phys. Rev. Lett. 115, 267203 (2015).

[41] G. J. Sreejith and S. Powell, Scaling dimensions of highercharge monopoles at deconfined critical points, Phys. Rev. B 92, 184413 (2015).

[42] P. Serna and A. Nahum, Emergence and spontaneous breaking of approximate $\mathrm{O}(4)$ symmetry at a weakly first-order deconfined phase transition, Phys. Rev. B 99, 195110 (2019).
[43] A. W. Sandvik and B. Zhao, Consistent scaling exponents at the deconfined quantum-critical point, Chin. Phys. Lett. 37, 057502 (2020).

[44] A. Pelissetto and E. Vicari, Multicomponent compact Abelian-Higgs lattice models, Phys. Rev. E 100, 042134 (2019).

[45] C. Bonati, A. Pelissetto, and E. Vicari, Lattice Abelian-Higgs model with noncompact gauge fields, arXiv:2010.06311.

[46] E. Fradkin and S. Shenker, Phase diagrams of lattice gauge theories with Higgs fields, Phys. Rev. D 19, 3682 (1979).

[47] A. Sudb $\varnothing$, E. Smørgrav, J. Smiseth, F. S. Nogueira, and J. Hove, Criticality in the (2+1)-Dimensional Compact Higgs Model and Fractionalized Insulators, Phys. Rev. Lett. 89, 226403 (2002).

[48] J. Smiseth, E. Smørgrav, F. S. Nogueira, J. Hove, and A. Sudbø, Phase structure of $d=2+1$ compact lattice gauge theories and the transition from Mott insulator to fractionalized insulator, Phys. Rev. B 67, 205104 (2003).

[49] F. S. Nogueira, J. Smiseth, E. Smørgrav, and A. Sudbø, Compact $U(1)$ gauge theories in $2+1$ dimensions and the physics of low-dimensional insulating materials, Eur. Phys. J. C 33, 885 (2004).

[50] M. N. Chernodub, R. Feldmann, E.-M. Ilgenfritz, and A. Schiller, The compact $Q=2$ Abelian Higgs model in the London limit: Vortex-monopole chains and the photon propagator, Phys. Rev. D 71, 074502 (2005).

[51] M. N. Chernodub, E.-M. Ilgenfritz, and A. Schiller, Phase structure of an Abelian two-Higgs model and high temperature superconductors, Phys. Rev. B 73, 100506(R) (2006)

[52] S. Wenzel, E. Bittner, W. Janke, and A. M. J. Schakel, Percolation of vortices in the 3D Abelian lattice Higgs model, Nucl. Phys. B 793, 344 (2008).

[53] M. E. Fisher and M. N. Barber, Scaling Theory for FiniteSize Effects in the Critical Region, Phys. Rev. Lett. 28, 1516 (1972).

[54] M. N. Barber, in Phase Transitions and Critical Phenomena, edited by C. Domb and J. L. Lebowitz (Academic Press, New York, 1983), Vol. 8.

[55] Edited by V. Privman, Finite Size Scaling and Numerical Simulation of Statistical Systems (World Scientific, Singapore, 1990).

[56] A. Pelissetto and E. Vicari, Critical phenomena and renormalization group theory, Phys. Rep. 368, 549 (2002).

[57] A. Pelissetto and E. Vicari, Three-dimensional ferromagnetic $\mathrm{CP}^{N-1}$ models, Phys. Rev. E 100, 022122 (2019).

[58] A. Pelissetto and E. Vicari, Large- $N$ behavior of threedimensional lattice $\mathrm{CP}^{N-1}$ models, J. Stat. Mech. (2020) 033209.

[59] M. Hasenbusch, Monte Carlo study of a generalized icosahedral model on the simple cubic lattice, Phys. Rev. B 102, 024406 (2020).

[60] M. V. Kompaniets and E. Panzer, Minimally subtracted six-loop renormalization of $\phi^{4}$-symmetric theory and critical exponents, Phys. Rev. D 96, 036016 (2017).

[61] M. Hasenbusch and E. Vicari, Anisotropic perturbations in 3D O(N) vector models, Phys. Rev. B 84, 125136 (2011).

[62] M. Campostrini, M. Hasenbusch, A. Pelissetto, P. Rossi, and E. Vicari, Critical exponents and equation of state of the threedimensional Heisenberg universality class, Phys. Rev. B 65, 144520 (2002). 
[63] R. Guida and J. Zinn-Justin, Critical exponents of the $N$-vector model, J. Phys. A 31, 8103 (1998).

[64] J. Hove and A. Sudbø, Criticality versus $q$ in the $(2+1)-$ dimensional $Z_{q}$ clock model, Phys. Rev. E 68, 046107 (2003).

[65] M. Hasenbusch, Monte Carlo study of an improved clock model in three dimensions, Phys. Rev. B 100, 224517 (2019).

[66] P. Patil, H. Shao, and A. W. Sandvik, Unconventional U(1) to $Z_{q}$ cross-over in quantum and classical q-state clock models, arXiv:2009.03249.

[67] A. M. Ferrenberg, J. Xu, and D. P. Landau, Pushing the limits of Monte Carlo simulations for the three-dimensional Ising model, Phys. Rev. E 97, 043301 (2018).

[68] H. G. Ballesteros, L. A. Fernandez, V. Martín-Mayor, and A. Muñoz Sudupe, Finite-size effects on measures of critical exponents in $d=3 O(N)$ models, Phys. Lett. B 387, 125 (1996).

[69] P. Butera and M. Comi, $N$-vector spin models on the sc and the bcc lattices: A study of the critical behavior of the susceptibility and of the correlation length by high temperature series extended to order $\beta^{21}$, Phys. Rev. B 56, 8212 (1997).

[70] F. Delfino, A. Pelissetto, and E. Vicari, Three-dimensional antiferromagnetic $\mathrm{CP}^{N-1}$ models, Phys. Rev. E 91, 052109 (2015).

[71] M. Campostrini, A. Pelissetto, P. Rossi, and E. Vicari, Fourpoint renormalized coupling in $\mathrm{O}(N)$ models, Nucl. Phys. B 459, 207 (1996).

[72] M. Moshe and J. Zinn-Justin, Quantum field theory in the large $N$ limit: A review, Phys. Rep. 385, 69 (2003).

[73] B. Ihrig, N. Zerf, P. Marquard, I. F. Herbut, and M. M. Scherer, Abelian Higgs model at four loops, fixed-point collision and deconfined criticality, Phys. Rev. B 100, 134507 (2019).
[74] K.-S. Liu and M. E. Fisher, Quantum lattice gas and the existence of a supersolid, J. Low Temp. Phys. 10, 655 (1972).

[75] M. E. Fisher and D. R. Nelson, Spin Flop, Supersolids, and Bicritical and Tetracritical Points, Phys. Rev. Lett. 32, 1350 (1974).

[76] D. R. Nelson, J. M. Kosterlitz, and M. E. Fisher, Renormalization-Group Analysis of Bicritical and Tetracritical Points, Phys. Rev. Lett. 33, 813 (1974); J. M. Kosterlitz, D. R. Nelson, and M. E. Fisher, Bicritical and tetracritical points in anisotropic antiferromagnetic systems, Phys. Rev. B 13, 412 (1976).

[77] P. Calabrese, A. Pelissetto, and E. Vicari, Multicritical behavior of $O\left(n_{1}\right) \oplus O\left(n_{2}\right)$-symmetric systems, Phys. Rev. B 67, 054505 (2003).

[78] F. Kos, D. Poland, D. Simmons-Duffin, and A. Vichi, Precision islands in the Ising and $\mathrm{O}(N)$ models, J. High Energy Phys. 08 (2016) 036.

[79] M. Hasenbusch, Finite-size scaling study of lattice models in the three-dimensional Ising universality class, Phys. Rev. B 82, 174433 (2010).

[80] We report here an accurate expression (deviations are assumed to be well below $0.5 \%$ ) of the universal curve $U=F\left(R_{\xi}\right)$ for the Heisenberg universality class: $F(x)=5 / 3+x(3.0263535+$ $23.139470 x)\left(1-e^{-15 x}\right)-47.838890 x^{2}+58.489668 x^{3}-$ $67.020681 x^{4}+38.408855 x^{5}-8.8557348 x^{6}$.

[81] T. A. De Grand and D. Toussaint, Topological excitations and Monte Carlo simulation of Abelian gauge theory, Phys. Rev. D 22, 2478 (1980).

[82] V. Yu. Irkhin, A. A. Katanin, and M. I. Katsnelson, $1 / N$ expansion for critical exponents of magnetic phase transitions in the $C P^{N-1}$ model for $2<d<4$, Phys. Rev. B 54, 11953 (1996). 